Running head: INDIVIDUAL TREATMENT EFFECTS WITH LATENT VARIABLES

Interindividual Differences in Treatment Effects based on Structural Equation Models with Latent Variables: An EffectLiteR Tutorial

\author{
Axel Mayer \\ RWTH Aachen University \\ Johannes Zimmermann \\ University of Kassel \\ Jürgen Hoyer \\ Technische Universität Dresden \\ Simone Salzer \\ International Psychoanalytic University Berlin \\ Jörg Wiltink \\ Johannes Gutenberg University Mainz \\ Eric Leibing \\ University of Göttingen \\ Falk Leichsenring \\ Justus Liebig University Giessen
}

This is a pre-copyedited, author-produced PDF of an article accepted for publication in Structural Equation Modeling: A Multidisciplinary Journal following peer review.

Citation:

Mayer, A., Zimmermann, J., Hoyer, J., Salzer, S., Wiltink, J., Leibing, E., \& Leichsenring, F. (in press). Interindividual differences in treatment effects based on structural equation models with latent variables: An EffectLiteR tutorial. Structural Equation Modeling.

Correspondence should be addressed to Axel Mayer, Department of Psychological Methods, Institute of Psychology, RWTH Aachen University, Jaegerstr. 17/19, D-52066 Aachen, Germany. Email: axel.mayer@rwth-aachen.de. Eric Leibing and Falk Leichsenring share last-authorship. 


\begin{abstract}
The investigation of interindividual differences in the effects of a treatment is challenging, because many constructs-of-interest in psychological research such as depression or anxiety are latent variables and modeling heterogeneity in treatment effects requires interactions and potentially nonlinear relationships. In this paper, we present a tutorial of the EffectLiteR approach (Mayer, Dietzfelbinger, Rosseel, \& Steyer, 2016) that allows for estimating individual treatment effects based on latent variable models. We describe step by step how to apply the approach using the EffectLiteR software package with data from the multicenter randomized controlled trial of the Social Phobia Psychotherapy Network (SOPHO-NET) and provide guidelines and recommendations for researchers. The focus of the paper is on explaining the results of a comprehensive effect analysis in an accessible language and on highlighting the opportunities the EffectLiteR approach offers for analyzing interindividual differences in treatment effects.
\end{abstract}

Keywords: Individual Treatment Effects; Structural Equation Modeling; Latent Variables; Moderation; Interaction; Causal Effects 


\section{Interindividual Differences in Treatment Effects based on Structural Equation Models with Latent Variables: An EffectLiteR Tutorial}

Traditionally, the majority of studies investigating the effectiveness of a treatment or an intervention focused on the average effect, i.e., whether there is an effect on average of a treatment compared to either a control group or compared to an alternative treatment with regard to a relevant outcome measure. The causal inference literature has long recognized the importance of interindividual differences in treatment effects and thus most causality theories start with defining an individual causal effect (Heckman \& Vytlacil, 2005; Holland, 1988; Manski, 1995; Pearl, 2009; Rubin, 1974; Steyer et al., 2000; Winship \& Morgan, 1999). Recently, more and more researchers across different disciplines started focusing on how the effectiveness of the treatment varies across persons in addition to the average effect: Examples come from political analysis (Imai \& Strauss, 2011), educational science (Beemer et al., 2018; Morgan, 2001), sociology (Xie, Brand, \& Jann, 2012), econometrics (Li \& Tobias, 2011), psychology (DeRubeis et al., 2014; Huibers et al., 2015; Simon \& Perlis, 2010), epidemiology (Dahabreh, Hayward, \& Kent, 2016), and medicine. Especially in the latter field, where the terms personalized medicine (Hamburg \& Collins, 2010) and precision medicine (Ashley, 2015) have been coined, there is a lot of attention nowadays for such interindividual differences in responses to treatments.

Researchers use a wide range of statistical methods to estimate individual effects and heterogeneity in individual treatment effects. Traditional ordinary least squares regression with interactions (i.e., moderation analysis; Aiken \& West, 1991) have been extended to include multiple moderators simultaneously and modern methods from the machine learning literature have been adopted (e.g., Athey \& Imbens, 2015, 2016; Imai \& Ratkovic, 2013; Wager \& Athey, 2017). However, none of the aforementioned approaches can incorporate latent variables, which are very common in the social and behavioral sciences (Bandalos, 2018). In this paper, we aim at closing this gap and present a tutorial of the EffectLiteR approach (Mayer, Dietzfelbinger, Rosseel, \& Steyer, 2016). This approach enables us to look at heterogeneity of effects and to identify the driving variables that moderate the treatment effects using a structural equation modeling approach with latent variables.

\section{Focus of this paper}

While the statistical details of the EffectLiteR approach have been presented in a series of recent publications (Mayer et al., 2016; Mayer, Nagengast, Fletcher \& Steyer, 2014; Mayer, Umbach, Flunger \& Kelava, 2017), the present paper describes step by step how to apply the approach (including a software application) and provides researchers with guidelines and recommendations. The focus of the paper is on explaining the results of a comprehensive effect 
analysis in an accessible language and on highlighting the opportunities the EffectLiteR approach offers for analyzing the differential effectiveness of a treatment or an intervention. We apply the EffectLiteR approach to a dataset from psychotherapy research and give detailed advice on how to conduct a comprehensive analysis of differential treatment effects. We also present graphical ways to get a more detailed picture about who benefits most from which treatment. The most important advantages of using the EffectLiteR approach over existing approaches are its ease of use, the possibility to include latent variables and higher-order interactions, the graphical effect plots and additional tools that help making sense of the effect analysis, and the option to obtain standard errors for conditional treatment effects, i.e., effects for subgroups or effects given certain values of covariates.

The paper is structured as follows: We start with giving some background on the randomized controlled trial (RCT) used for illustration in this paper. Next, we introduce the most important concepts for analyzing heterogeneity of treatment effects. In this section we provide formal definitions of individual, aggregated/conditional, and average effects and mention causality conditions under which such effects can be estimated. We proceed with discussing the importance of latent variables and different ways how to include latent variables and interactions in the analysis. Then we present a comprehensive effect analysis using the EffectLiteR approach that can deal appropriately with these challenges. We show how EffectLiteR can be used to gain insight into which therapy is optimally suited for a particular patient. In the discussion, we give hints to additional features in EffectLiteR.

\section{The SOPHO-NET treatment study}

In this tutorial, we use data from the treatment study of the Social Phobia Psychotherapy Network (SOPHO-NET; Leichsenring et al., 2009, 2013, 2014) for illustrating a comprehensive effect analysis with a particular focus on examining interindividual differences in treatment effects based on structural equation models with latent variables. The SOPHO-NET treatment study is a multicenter RCT designed to investigate the effectiveness of cognitive-behavioral therapy (CBT) and short-term psychodynamic psychotherapy (STPP) for treatment of social phobia. The primary outcome measure used in this study is the Liebowitz Social Anxiety Scale (LSAS; Liebowitz, 1987), a clinician-administered rating scale assessing the severity of social anxiety disorder. The SOPHO-NET trial is a longitudinal study with multiple occasions of measurement. For illustration, we only use data from the pre-treatment and post-treatment assessments (Leichsenring et al., 2013).

The SOPHO-NET trial is especially suited for demonstrating the EffectLiteR approach because it features a relatively large sample size (with 209 patients being randomized to CBT and 207 patients being randomized to STPP) as well as a comprehensive battery of measures that were applied at baseline. Besides the LSAS as the primary outcome measure this battery includes, for 
example, the Structured Clinical Interview for DSM-IV Axis I (First, Spitzer, Gibbon, \& Williams, 2002) and Axis II (First, Gibbon, Spitzer, Williams, \& Benjamin, 1997) disorders assessing comorbid mental disorders, the Tridimensional Personality Questionnaire (TPQ; Cloninger, 1987) assessing the personality dimensions novelty seeking, harm avoidance and reward dependence, the Inventory of Interpersonal Problems_-Circumplex (IIP-C; Alden, Wiggins, \& Pincus, 1990) assessing the severity and style of interpersonal problems (including the dimensions of dominance and affiliation), the Experiences in Close Relationships - Revised (ECR-R; Fraley, Waller, \& Brennan, 2000) questionnaire assessing anxious and avoidant attachment styles, the Frankfurt Self Concept Scales (FSCS; Deusinger, 1986) assessing different facets of the self-concept (including self-esteem), the Beck Depression Inventory (BDI; Beck, Ward, Mendelson, Mock \& Erbaugh, 1961) assessing the severity of depressive symptoms, and the Test of Self-Conscious Affect (TOSCA; Tangney, Wagner, \& Gramzow, 1989) assessing shame and guilt proneness. In previous publications based on the SOPHO-NET trial these measures have already been investigated as predictors of treatment outcome (Hoyer et al., 2016; Wiltink et al., 2016) or (in part) as moderators of the treatment effect (Strauss et al., 2017) using multiple regression analyses.

\section{Individual and Conditional Treatment Effects}

Definitions of individual treatment effects can be found in the causal inference literature (e.g., Imbens \& Rubin, 2015; Rubin, 1974; Pearl, 2009; Steyer, Mayer \& Fiege, 2014). The key idea of defining an individual treatment effect of a treatment $X=1$ vs. another treatment $X=0$ is to compare the expected outcome under both treatment conditions for a concrete person ${ }^{1}$. For a patient in the SOPHO-NET study, interest lies in comparing his/her expected social anxiety level if treated with STPP to his/her expected social anxiety level if treated with CBT. More formally, we define the expected outcome for person $U=u$ under treatment $X=1$ as $E(Y \mid X=1, U=u)$ and the expected outcome for person $U=u$ under treatment $X=0$ as $E(Y \mid X=0, U=u)$. The individual treatment effect is then defined as the difference between the two person-specific expected outcomes ${ }^{2}$, i.e.,

$$
\delta_{10}(u)=E(Y \mid X=1, U=u)-E(Y \mid X=0, U=u) .
$$

Ideally, we would want to directly estimate the individual treatment effects in an empirical study. Unfortunately, this is not possible in most cases due to the problem known as the fundamental problem of causal inference (Holland, 1986). The fundamental problem of causal inference describes the fact that a person can only be observed once under one of the treatment condition and never simultaneously in all treatment conditions. In an ideal world, we would be able to observe

1 In many studies one of the two treatment conditions is an untreated control group (e.g., $X=c)$ but in this paper we use the theory to compare the (differential) effectiveness of two competing treatments.

2 We use stochastic expected outcomes notation (following Steyer's theory of causal effects) instead of the more frequently used potential outcomes notation (according to Rubin's theory). Stochastic expected outcomes assume an intra-individual distribution under each treatment condition and consider the expected value of this intra-individual distribution instead of a fixed value for a potential outcome. See Steyer (2005) for a discussion about the stochastic nature of true outcomes. For the data analysis presented later in this article, the two theories have similar implications. 
every person multiple times under each of the treatment conditions. Only then would we be able to directly estimate the intra-individual distribution under each treatment and obtain unbiased estimates for the individual treatment effects as defined above.

Since the individual treatment effects and the expected outcomes for each person under both treatment conditions cannot be observed, we try to get as close as possible to these effects by identifying all covariates that explain unique variance in the outcome variable conditional on the treatment and on other covariates. Steyer et al. (2000) term this causality condition conditional unittreatment homogeneity. Of course, "all covariates" is a strong and often unrealistic claim but when we are interested in individual effects we need to make every effort to get as close to this ideal as possible. In the discussion, we describe some potential problems with covariate selection and give hints to approaches that aid in the selection process. Once we have identified all relevant covariates $Z$, we consider the conditional treatment effects given these covariates defined as $\mathrm{CE}_{10}(z)=E(Y \mid$ $X=1, Z=z)-E(Y \mid X=0, Z=z)$. If conditional unit-treatment homogeneity holds, the conditional treatment effects $\mathrm{CE}_{10}(z)$ are equal to the individual causal effects $\delta_{10}(U)$ and can be estimated based on data from an empirical study ${ }^{3}$. Furthermore, once we have identified the conditional treatment effects $\mathrm{CE}_{10}(z)$, we can again aggregate and consider aggregated effects at a higher level, such as effects for subgroups (males and females; older and younger patients) or effects given a subset of all relevant covariates. We define these aggregated effects by $\left.\mathrm{E}_{\mathrm{CE}} \mathrm{CE}_{10}(z) \mid W=w\right]$, where $W$ is a random variable indicating subgroup or a subset of all covariates $Z$. In traditional RCTs, interest lies in the average effect in the population defined as the unconditional expectation of the conditional effects, $\mathrm{AVE}_{10}=E\left[\mathrm{CE}_{10}(z)\right]$, or in aggregated effects for subgroups.

\section{Heterogeneous Treatment Effects in Psychotherapy Research}

In psychotherapy research, average effects comparing different treatments (e.g., psychotherapy and medication, or different psychotherapeutic treatments) are oftentimes small or not significant (Wampold \& Imel, 2015). For example, in the SOPHO-NET trial, analysis of the manifest test scores of the primary outcome measure (LSAS) revealed a significant average effect at post-treatment in favor of CBT, but this effect was small, with an effect size of $d=0.25,95 \% \mathrm{CI}$ [0.06, 0.44] (Leichsenring et al., 2013). So recently, attention has shifted and there is much interest and emphasis in the estimation of interindividual differences in treatment effects in addition to considering the average effect (DeRubeis et al., 2014; Fournier et al., 2009; Kazdin, 2014; but see Snow, 1991, for an early emphasis). Learning about such interindividual differences is a promising way to optimize the therapeutic indication and thereby the efficacy of the treatment for the individual patient and on average (Cohen \& DeRubeis, 2018, Kessler, 2018). The question of who

3 The empirical study can be a RCT, but does not have to be randomized, because conditional unit-treatment homogeneity is a sufficient condition for obtaining conditional causal effects even in observational studies (see Steyer et al. 2000). 
benefits most from a specific treatment has been addressed from multiple approaches (e.g., Delgadillo et al., 2016; Driessen et al., 2016; Niles et al., 2017), and one of the most well-known approaches is the so-called personalized advantage index (PAI; Deisenhofer et al., 2018; DeRubeis et al., 2014; Huibers et al., 2015; Webb et al., in press). The PAI is the result of calculating the difference of the expected outcomes (e.g., the expected post-treatment depression) of two different therapies for each person. The expected outcomes are estimated based on a regression model and the sign of the PAI indicates which therapy is preferred. Thus, the PAI represents a specific approach of estimating individual treatment effects.

\section{Latent Variables in Estimating Interindividual Differences in Treatment Effects}

In psychological research and the social sciences in general the constructs-of-interest are oftentimes latent variables and cannot be observed directly. Many constructs like depression or anxiety are measured using multiple fallible indicators such as items in a questionnaire. The latent constructs can be both covariates or outcomes in the analysis and it may be challenging to formulate proper measurement models for these latent variables. For example, in the SOPHO-NET study the primary outcome measure is the latent post-treatment social anxiety assessed by the items in the LSAS and there are multiple latent covariates including baseline measures of social anxiety, depression, personality, interpersonal problems and others (see above). We can in principle use four different strategies to deal with these latent constructs: We can either use the mean (total) test scores, or we can compute factor scores for the latent variables before the analysis, or we can construct item parcels and use these parcels in the measurement model, or we can use all items in a measurement model for the latent construct. Using the total test scores or factor scores results in single indicators of a construct that do not allow for separating measurement error variance from true score variance. It is well-known that ignoring measurement error can lead to bias in regression coefficients and this is particularly problematic for interaction terms (e.g., Jaccard \& Wan, 1995). Thus, measurement by multiple indicators calls for more sophisticated models in the context of assessing heterogeneous treatment effects where interactions are crucial. Using all items or using item parcels in appropriate measurement models are two possibilities to account for measurement error (see Little et al., 2013, and Marsh et al., 2013, for a comprehensive scientific discourse about the pros and contras of both strategies). An item parcel is an aggregated indicator comprised of the average of two or more items. In the context of estimating interindividual differences in treatment effects, we typically need to include many latent variables in our analysis model, which makes it computationally difficult to use all items in the measurement models. In the SOPHO-NET study, social anxiety is measured by 48 items (LSAS), depression is measured by 21 items (BDI), 
attachment avoidance is measured by 18 items (ECR-R) and so forth. The focus of this paper is not on evaluating the psychometric properties of these questionnaires which have been well established. Thus, to keep our analysis feasible and still account for measurement error, we decided to use item parcels in our models. Item parcels are a viable alternative and require estimation of far less parameters (see Table 3 in Little et al., 2013 for a full list of advantages of item parcels).

\section{Covariate Selection and Interactions}

Another challenge in an analysis of interindividual differences in treatment effects is how to select the covariates. The covariate selection process should ideally be guided by theoretical and statistical considerations. Only when all relevant covariates are included in the selection can we model all the heterogeneity in treatment effects and assess the relative roles of each of the covariates. In almost all cases, we need to consider multiple covariates (sometimes also called potential moderators) simultaneously to get an adequate picture of variability in treatment effects. One of the key questions applied researchers are typically faced with is which covariates should be included and which not. This is a difficult question and there is no simple answer or strategy to this question that is always right. Substantive theories, careful thinking, experience from previous research, statistical tools, and a solid understanding of the limitations of statistical models play an important role in the process. In our analysis of the SOPHO-NET study, we are in the fortunate situation that we can rely on findings from previous research that has examined predictors of outcomes in detail (Hoyer et al., 2016; Wiltink et al., 2016). We will provide more details about the selection process in Step 4 of our description of the EffectLiteR analysis. In situations where less is known about covariates that predict the outcome of interest, there are many statistical tools that can assist in the selection process. We mention some of these tools along with some references in the discussion.

The correct specification of interactions and the functional form of the regression is another challenge when modeling heterogeneous treatment effect that is closely intertwined with the covariate selection process. Once we have identified potentially relevant covariates, we need to carefully consider which interactions and other non-linear terms we need in order to model the conditional expectations appropriately. It is crucial to incorporate flexibility in the regression model by allowing for interactions and potentially non-linear relationships. Two-way or higher-order interactions between the treatment variable and one or multiple covariates usually play an important role, when the goal is to assess interindividual differences in treatment effects, because omitting relevant interactions may lead to an underestimation of the variance of treatment effects.

With our EffectLiteR approach it is straightforward to include interactions between the treatment variable and categorical and continuous (latent) covariates, because it uses a multigroup structural equation model for the estimation of parameters. In some applications it is necessary to 
include higher-order interactions (e.g., Flunger, Umbach \& Mayer, 2018) or interactions with quadratic terms in the model (Mayer, Umbach, Flunger, \& Kelava, 2017). In our analysis of the SOPHO-NET study, we combine the covariate selection process and the inclusion of interactions and simultaneously test for effects and interactions between covariates and the treatment using overall hypothesis tests (see Step 5 of our EffectLiteR analysis for details).

\section{EffectLiteR Analysis of Individual Treatment Effects}

We now describe our EffectLiteR analysis of the SOPHO-NET study step-by-step. The EffectLiteR approach for analyzing conditional effects based on structural equation modeling has recently been developed (Mayer, Dietzfelbinger, Rosseel \& Steyer, 2016), building on earlier work by Partchev \& Steyer (2008). The two most prominent strengths of this approach in this context are the possibility to include latent variables in an effect analysis and to obtain standard errors for conditional effects. EffectLiteR is both the name of the approach for analyzing differential treatment effects and the name of a corresponding software package that makes the approach easily accessible for applied researchers. EffectLiteR is a R package (R Core Team, 2012) with a convenient graphical user interface based on the shiny package (Chang, Cheng, Allaire, Xie \& McPherson, 2018) and can also be used without any knowledge in $R$ and even without ever seeing the $R$ command line. The graphical user interface allows for convenient specification of the model and includes several graphical illustrations to explore the differential advantages of the treatments. By relying on the open source SEM software lavaan (Rosseel, 2012), we are also able to use many modern statistical techniques like robust estimators, full information maximum likelihood estimation for missing data or models with stochastic regressors in EffectLiteR. In this paper, we show step by step how to install the program, how to specify the input and how to interpret the results of a comprehensive analysis of individual treatment effects with latent variables using a high quality dataset from the SOPHO-NET study.

\section{Step 1: Install EffectLiteR}

There are multiple ways how to install the software package: Windows users without experience in $\mathrm{R}$ who only want to use the graphical user interface can download the Windows installer (www.causal-effects.de) and follow the instructions. If we want to use both, the command line interface and the graphical user interface, we first need to install R and then copy-and-paste the following three lines into the command line:

install.packages("EffectLiteR")

library(EffectLiteR)

effectLiteGUI() 
The first command installs the package, the second command loads the package and the third command opens the graphical user interface in the system default browser. Since R itself is crossplatform this works on all major operating systems (Windows, MacOS, Linux). Note that even though EffectLiteR uses a browser to show the graphical user interface, it runs locally, i.e., it does not need an internet connection and never uploads any data or any other information (which is important for usage with confidential data). If the installation was successful, we then see a browser window with the name EffectLiteR on top and an input panel on the left-hand side (with tabs: data, manifest variables, latent variables and so forth) and an output panel on the right-hand side (with tabs: data, EffectLiteR, syntax, results and so forth). This tutorial uses the graphical user interface, but we also provide code for the command line interface in Appendix A.

\section{Step 2: Read data and options}

At first, we need to open a dataset. We can either use one of the built-in datasets for teaching purposes or we can read-in an external dataset by clicking on the "Browse" button in the data input panel and selecting the data file stored on the computer. The graphical user interface accepts a wide range of data types (.csv, .dat, .txt, .sav, .xpt or .rds files) and tries to automatically select the appropriate R function to read-in the dataset. In most test cases, including the SPSS file with the SOPHO-NET data, this works out of the box. The user can specify additional options in case there are problems with the default settings. The analyses in this manuscript are conducted with the original SOPHO-NET data. Researchers interested in using this data can apply with the SOPHONET consortium. For teaching purposes, we included a simulated dataset that is similar to the original data ${ }^{4}$ in the EffectLiteR package (see Appendix A for the code to use this simulated data; it can also be accessed directly in the example data drop-down menu in the graphical user interface). After reading the data file successfully, we see the first 25 rows of the data table in the data output panel on the right-hand side. Here we can check the data and also request to see the remaining rows.

Since there are some missing values in our dataset, we go to the options input panel and select fiml before running the analysis. Thereby, the analysis uses full information maximum likelihood (FIML, Enders \& Bandalos, 2001) instead of the default setting listwise deletion to better handle missing data.

4 The simulated dataset is generated using the group-specific model implied variance-covariance matrix and mean vector of the final analysis model. No real patient data is included. This way the point estimates of the conditional and aggregated effects based on the simulated data match with the ones presented based on the original data used in this manuscript. There can be slight differences in indiviudal treatment effects and standard errors. There are no missing values in the simulated dataset and therefore the sample size was adjusted to yield similar standard errors. If the model is fit with the simulated dataset, the model fit will be perfect, because the model implied statistics have been used to generate this data. 


\section{Step 3: Specify manifest and latent variables}

The key part of the model specification is to select the variables that we want to include in the analysis. There are two sections in the input panel, one for manifest variables and one for latent variables. In our analysis of the SOPHO-NET data, we start with the full model including all variables for which previous research has shown a significant bivariate relationship with the outcome in either the CBT group (Hoyer et al., 2016) or the STPP group (Wiltink et al., 2016). Under manifest variables, we specify the manifest treatment variable (treat) indicating cognitive behavioral therapy (CBT) and short-term psychodynamic psychotherapy (STPP) and the three manifest continuous covariates, including the test scores for dominance and affiliation (according to IIP-C) and the number of comorbid mental disorders (according to SCID-I and -II). For all other variables, we created item parcels and model them as latent variables (see below). We did not create item parcels for the IIP-C test scores, because the latent structure of this measure is more complex and does not easily allow for building item parcels (Alden et al., 1990). There are no categorical covariates in this analysis and the dependent variable is latent, so we leave these two fields empty in the manifest variables input panel. The left-hand side of Figure 1 shows the graphical user interface for the manifest variables.

Next, we go to the latent variable input panel and click on the checkbox indicating that the dependent variable is latent. Then we see a new input field that allows us to select the manifest indicators for the dependent variable from the list of variables in our dataset (see right-hand side of Figure 1). The shiny interface uses fuzzy matching of variable names, which means that we can just start typing (parts of) the variable name and we get suggestions. This is especially helpful in large datasets such as the SOPHO-NET study. In our analysis, we use two item parcels (1sas.a.t2 and lsas.v.t2) as indicators for the latent dependent variable post-treatment social anxiety: The first indicator is the mean score of the 24 anxiety items and the second indicator is the mean score of the 24 avoidance items at the post-treatment time point. The program automatically specifies a measurement model for the latent variable in the background. By default, it uses a group-invariant tau-equivalent measurement model for latent variables with two indicators, i.e., a measurement model where both loadings are set to one, the intercepts of the first indicator are set to zero and the intercepts of the second indicator are estimated but constrained to be equal across treatment groups (Meredith, 1993). For latent variables with more than two indicators, the default setting is to use a group-invariant tau-congeneric measurement model. In most cases, these default settings are a reasonable choice but the user can have full control and specify any identified measurement model if needed.

After having specified the latent dependent variable, we next tell the program how many latent covariates we want to have in our analysis. For each latent covariate the user interface 
displays an input field where we can specify the indicators from the list of variables in the dataset. We include seven different latent covariates in our full model: Latent variables for the pre-treatment severity of social anxiety (LSAS), the severity of depressive symptoms (BDI), the personality dimension harm avoidance (TPQ), attachment anxiety and avoidance (ECR-R), self-esteem (FSCN) and shame (TOSCA). For the pre-treatment social anxiety we use the same indicators as for the post-treatment measurement. For all other latent covariates, we created two (self-esteem and shame) or three item parcels (depression, harm avoidance, attachment anxiety, attachment avoidance) for each latent variable using an odd-even allocation of items to parcels. The item parcels were created in a preparatory data manipulation step before opening the EffectLiteR program. We use the default setting of a group-invariant measurement model for all latent variables in the model. Notice that the seven latent covariates are added to the list of three manifest covariates, so that our full model includes ten covariates.

\section{Step 4: Select covariates and interactions}

The specification of the full model is now complete and EffectLiteR automatically estimates the parameters of the full model in the background - we don't need to press a button. When we click on the EffectLiteR tab in the output panel we see a message on top that the model has converged successfully and that we obtain results. As mentioned above, we can rely on previous research for our covariate selection and do not want to delete covariates or interactions in the model. In settings where less is known about relevant covariates, EffectLiteR includes a tool to check if we actually need all covariates in the analysis. To use this tool, we can go to the userspecified tests tab in the input panel on the left-hand side and on the output panel, we also go to the user-specified tests tab. In the output tab we get a message that no subset of continuous covariates is specified, i.e., there is no test, yet. Assume for the sake of illustrating the covariate selection mechanism, we would like to test if we actually need attachment anxiety and avoidance in the model. In statistical terms, we test the joint null hypothesis that the regression coefficients (main effects and interactions involving one of them) related to these two latent variables are zero. In order to test this particular hypothesis, we select the two latent variables in the input panel under the heading "Test continuous covariates". In the output panel on the right-hand side we then get a Wald test statistic for the hypothesis that the selected covariates have no effects (Wald $\chi^{2}=10.62, d f=4$, $p=0.031$ ). Since the joint hypothesis test is significant, we decide not to omit the two attachment latent variables which is in line with the findings from previous research (Strauss et al., 2017).

\section{Step 5: Main output and hypotheses}

After having specified the final model for our analysis, we are ready to look at some more details and first go to the main output which is shown in the tab with the name EffectLiteR in the 
output panel on the right-hand side. First we get a summary of the input specification with the variable names that are included in the analysis. In the main output the letter $Y$ is used for the latent dependent variable $\eta, X$ is used for the treatment variable and $Z=\left(Z_{1}, Z_{2}, Z_{3}, Z_{4}, Z_{5}, Z_{6}, Z_{7}, Z_{8}, Z_{9}\right.$, $\left.Z_{10}\right)$ is used for the vector of the three manifest covariates $\left(Z_{1}=\right.$ comorbid mental disorders; $Z_{2}=$ IIP affiliation; $Z_{3}=$ IIP dominance $)$ and the seven latent covariates $\left(Z_{4}=\xi_{1}=\right.$ pre-treatment LSAS; $Z_{5}=$ $\xi_{2}=\mathrm{BDI} ; Z_{6}=\xi_{3},=\mathrm{ECR}$ anxiety; $Z_{7}=\xi_{4}=\mathrm{ECR}$ avoidance; $Z_{8}=\xi_{5}=$ TPQ harm avoidance; $Z_{9}=\xi_{6}$ $=$ TOSCA shame; $Z_{10}=\xi_{7}=$ FSCN self-esteem). The treatment variable has two levels (CBT and STPP) and CBT is automatically selected as the reference group. The reference group can be changed in the options tab in the input panel if needed. Then we see the regression model in equation form and the corresponding point estimates, standard errors and $p$-values (see Table 1). In order to analyze differential treatment effects we consider all interactions between covariates and the treatment variable, which is the default in EffectLiteR. The model equations for our analysis are:

$$
\begin{aligned}
E(Y \mid X, Z)= & g_{0}(Z)+g_{1}(Z) \cdot X \\
g_{0}(Z)= & \gamma_{00}+\gamma_{01} \cdot Z_{1}+\gamma_{02} \cdot Z_{2}+\gamma_{03} \cdot Z_{3}+\gamma_{04} \cdot \xi_{1}+\gamma_{05} \cdot \xi_{2}+\gamma_{06} \cdot \xi_{3}+\gamma_{07} \cdot \xi_{4}+ \\
& +\gamma_{08} \cdot \xi_{5}+\gamma_{09} \cdot \xi_{6}+\gamma_{010} \cdot \xi_{7} \\
g_{1}(Z)= & \gamma_{10}+\gamma_{11} \cdot Z_{1}+\gamma_{12} \cdot Z_{2}+\gamma_{13} \cdot Z_{3}+\gamma_{14} \cdot \xi_{1}+\gamma_{15} \cdot \xi_{2}+\gamma_{16} \cdot \xi_{3}+\gamma_{17} \cdot \xi_{4}+ \\
& +\gamma_{18} \cdot \xi_{5}+\gamma_{19} \cdot \xi_{6}+\gamma_{110} \cdot \xi_{7}
\end{aligned}
$$

Inserting the equation for the $g_{0}(Z)$ and $g_{1}(Z)$ functions into the first Equation reveals that we are estimating a model with interactions between the intervention and all covariates in this general moderated regression model. Following Mayer et al. (2016), we write the regression model with an intercept function $g_{0}(Z)$ and an effect function $g_{1}(Z)$. The intercept function represents the effects of the covariates in the reference group CBT. The values of the effect function $g_{1}(Z)$ represent the conditional treatment effects $g_{1}(Z)=\mathrm{CE}_{10}(Z)$ comparing STPP to CBT given particular values of the covariates $\mathrm{Z}$. The underlying statistical model is described in the next section in an excursus for the interested reader.

In the main output we see a section labeled main hypotheses where four omnibus hypothesis tests are shown. From the perspective of estimating interindividual differences in treatment effects, the most interesting hypothesis is the third hypothesis "No treatment*covariate interactions". This is a global test that checks for any treatment by covariate interactions, in other words, it tests whether the treatment effects depend on values of covariates. If the treatment effects would not differ between values of covariates, the effect function $g_{1}(Z)$ was constant. Consequently, we test simultaneously whether all interaction terms are zero, i.e., whether all coefficients in $g_{1}(Z)$ except for the first one are zero. In our final model for the analysis of the SOPHO-NET dataset, the test statistic for the third hypothesis is significant (Wald $\chi^{2}=26.40, d f=10, p=0.003$ ) indicating that there are differences in the effectiveness of CBT and STPP depending on values of the ten 
covariates in the model. Since we find evidence for interindividual differences in treatment effects we now want to explore these differences in more detail.

\section{Excursus: Statistical model and computation of the effect function}

The underlying statistical model for our effect analysis is a multigroup structural equation model with stochastic group sizes. The multigroup SEM approach is necessary to include interactions between the treatment variable and the latent covariates. In our case we regress $\eta$ on the manifest covariates $Z_{1}$ to $Z_{3}$ and the latent covariates $\xi_{1}$ to $\xi_{7}$ in both the CBT group and the STPP group. A path diagram of this model is shown in Figure 2. The measurement model, the structural model, and the model for the group sizes are given by:

$$
\begin{aligned}
& \left(\begin{array}{c}
Y_{1} \\
Y_{2} \\
X_{11} \\
X_{21} \\
\vdots \\
X_{17} \\
X_{27}
\end{array}\right)=\left(\begin{array}{c}
0 \\
v_{20} \\
0 \\
v_{21} \\
\vdots \\
0 \\
v_{27}
\end{array}\right)+\left(\begin{array}{cccc}
1 & 0 & \cdots & 0 \\
1 & 0 & \cdots & 0 \\
0 & 1 & \cdots & 0 \\
0 & 1 & \cdots & 0 \\
\vdots & \vdots & \vdots & \vdots \\
0 & 0 & \cdots & 1 \\
0 & 0 & \cdots & 1
\end{array}\right) \cdot\left(\begin{array}{c}
\eta \\
\xi_{1} \\
\vdots \\
\xi_{7}
\end{array}\right)+\left(\begin{array}{c}
\epsilon_{10} \\
\epsilon_{20} \\
\epsilon_{11} \\
\epsilon_{21} \\
\vdots \\
\epsilon_{17} \\
\epsilon_{27}
\end{array}\right) \\
& \left(\begin{array}{c}
\eta \\
Z_{1} \\
Z_{2} \\
Z_{3} \\
\xi_{1} \\
\vdots \\
\xi_{7}
\end{array}\right)=\left(\begin{array}{c}
\alpha_{x 0} \\
\mu_{x 1} \\
\vdots \\
\mu_{x 7} \\
\mu_{x 8} \\
\mu_{x 9} \\
\mu_{x 10}
\end{array}\right)+\left(\begin{array}{ccccccc}
0 & \alpha_{x 1} & \cdots & \alpha_{x 7} & \alpha_{x 8} & \alpha_{x 9} & \alpha_{x 10} \\
0 & 0 & \cdots & 0 & 0 & 0 & 0 \\
\vdots & \vdots & \vdots & \vdots & \vdots & \vdots & \vdots \\
0 & 0 & \cdots & 0 & 0 & 0 & 0 \\
0 & 0 & \cdots & 0 & 0 & 0 & 0 \\
0 & 0 & \cdots & 0 & 0 & 0 & 0 \\
0 & 0 & \cdots & 0 & 0 & 0 & 0
\end{array}\right) \cdot\left(\begin{array}{c}
\eta \\
Z_{1} \\
Z_{2} \\
Z_{3} \\
\xi_{1} \\
\vdots \\
\xi_{7}
\end{array}\right)+\left(\begin{array}{c}
\xi_{0} \\
\xi_{1} \\
\vdots \\
\xi_{7} \\
\xi_{8} \\
\xi_{9} \\
\xi_{10}
\end{array}\right)
\end{aligned}
$$

Based on the group-specific regression coefficients $\alpha_{\mathrm{x} 0}$ to $\alpha_{\mathrm{x} 10}$, the program EffectLiteR computes the coefficients of the effect function (Equation 3) as follows:

$$
\begin{array}{lcl}
\gamma_{10}=\alpha_{10}-\alpha_{00} & \gamma_{11}=\alpha_{11}-\alpha_{01} & \gamma_{12}=\alpha_{12}-\alpha_{02} \\
\gamma_{13}=\alpha_{13}-\alpha_{03} & \gamma_{14}=\alpha_{14}-\alpha_{04} & \gamma_{15}=\alpha_{15}-\alpha_{05} \\
\gamma_{16}=\alpha_{16}-\alpha_{06} & \gamma_{17}=\alpha_{17}-\alpha_{07} & \gamma_{18}=\alpha_{18}-\alpha_{08} \\
\gamma_{19}=\alpha_{19}-\alpha_{09} & \gamma_{110}=\alpha_{110}-\alpha_{010} &
\end{array}
$$

The coefficients of the effect function are then key to exploring interindividual differences in effects as will be shown in the remainder of this article. The other parameters such as the group-specific means of the covariates $\mu_{\mathrm{x} 1}$ to $\mu_{\mathrm{x} 10}$ and the stochastic group weights $\kappa_{\mathrm{x}}$ are needed to compute average effects and some conditional effects such as effects on the treated which are not the focus of this article. 


\section{Step 6: Conditional effects given values of covariates}

We could take a look at the regression coefficients themselves (see Table 1), but they are difficult to interpret, especially because there are multiple correlated covariates and interaction terms in the analysis. A more instructive way to consider conditional treatment effects is to use the statistical model to get estimates of the individual effects. Recall that we need to assume unittreatment homogeneity, i.e., that there is no unobserved heterogeneity in treatment effects. Under this condition, we (just) need to know the relevant covariate values for a given person and can get an estimate for his/her individual effect by inserting theses values into the effect function $g_{1}(Z)$. The values of the effect function represent the difference between the expected outcome under STPP vs. the expected outcome under CBT for a person with the given covariate values. Formally, this can be written as $\mathrm{g}_{1}(Z)=\mathrm{CE}_{10}(Z)=E(Y \mid X=\mathrm{STPP}, Z)-E(Y \mid X=\mathrm{CBT}, Z)$, and the values of this function are equal to the individual effects if the unit-treatment homogeneity assumption holds. When the sign of the conditional effect is positive for a person it means that his/her expected social anxiety level is higher under STPP than under CBT, so for this person CBT is more effective. Equivalently, when the sign of the conditional effect is negative, it means that the expected social anxiety level is higher under CBT than under STPP, so in this case STPP is more effective.

EffectLiteR has a convenient interface to make it easier to look at various conditional effects given covariate values. To use this interface we go to the tab labeled "Conditional Effects II" in the output panel (see Figure 3). All ten covariates are listed on the left-hand side and the mean value is pre-specified for each of them. To give an idea about the scale of the continuous variables in the model the means and the standard deviations are shown, too (see Table 2). Notice that we fixed the first loading for each latent variable to 1 . Consequently, the scale of the latent variable is equal to the scale of the first item parcel, which itself is a mean score. For LSAS and BDI it is a mean score of 4-point Likert items with values 0 to 3, for ECR-R anxiety and avoidance it is a mean score of 7point Likert items with values 1 to 7 , for TPQ scales it is a mean score of binary items with values 0 and 1 , for TOSCA it is a mean score of 5-point Likert items with values 1 to 5 , and for FSCN it is a mean score of 6-point Likert items with values 1 to 6 . So if the values of the latent variable are unknown we can use the mean test score as an estimate. More sophisticated ways to estimate values of latent variables include factor score approaches (e.g., Devlieger, Mayer, \& Rosseel, 2015), which will be discussed later.

The main part of this output is the table labeled conditional effects and includes four columns. $g 1$ stands for the value of the effect function $g_{1}(Z=z)$ at the given covariate values $z^{5}$. Currently, it is an estimate of the treatment effect for an average person, i.e., a person with average covariate values. For such an average person, the estimated treatment effect is positive (Estimate $=$

5 We could calculate this value by hand, when we use the regression coefficients of the effect function in Table 2 and multiply them with the corresponding covariate values. 
$0.176, \mathrm{SE}=0.044)$ indicating an advantage of CBT. The value in the column labeled se_g1 is the corresponding standard error for this estimate. ExpOutc0 and ExpOutc1 represent the expected outcomes for this person under CBT and STPP. More formally, ExpOutc $0=E(Y \mid X=\mathrm{CBT}, Z=z)$ and ExpOutc1 $=E(Y \mid X=\mathrm{STPP}, Z=z)$. The difference between the two expected outcomes is equal to the conditional treatment effect for this average person. We can see that the expected LSAS post value for an average person is 0.969 under CBT and 1.145 under STPP which is essentially the same information as $\mathrm{g} 1$ and shows that CBT is expected to be more effective.

Now, we can start exploring different conditional effects by plugging in other reasonable values for the ten covariates. We give two more examples: First, we consider a person with one comorbid mental disorder $Z_{1}=1$, a very low IIP affiliation score $Z_{2}=-0.8$, relatively low scores for pre-treatment $\operatorname{LSAS}\left(\xi_{1}=0.8\right)$ and $\operatorname{BDI}\left(\xi_{2}=0.3\right)$, and average values on the other covariates. For this person we find a significant advantage of $\operatorname{STPP}(\mathrm{g} 1=-0.49, \mathrm{SE}=0.19, \operatorname{ExpOutc} 0=0.91$, ExpOutc1=0.42). Second, we consider a person with two comorbid mental disorders $Z_{1}=2$, high IIP affiliation score $Z_{2}=0.5$, relatively high scores for pre-treatment LSAS $\left(\xi_{1}=2\right)$ and BDI $\left(\xi_{2}=\right.$ 1 ), and average values on the other covariates. For this person we find a significant advantage of CBT $(\mathrm{g} 1=0.53, \mathrm{SE}=0.12$, ExpOutc0=1.02, ExpOutc1=1.55). So depending on the covariate values either STPP or CBT can have an advantage.

\section{Step 7: Heterogeneity of treatment effects in the sample}

In the next step, we would like to learn more about the heterogeneity of treatment effects in our sample. In order to estimate individual treatment effects for all persons in our sample we need estimates for the values of the latent covariates that we can insert into our effect function. As previously mentioned we could use the mean test scores as estimates or more sophisticated factor score approaches. By default EffectLiteR uses regression factor scores to obtain values of latent variables. Note that we neither use factor scores to estimate the parameters of the EffectLiteR model, nor the parameters of the conditional effects function nor the average and conditional effects in the main output. All this is based on the full latent variable multigroup structural equation model. Only for estimating heterogeneity of treatment effects in our sample we do need the factor scores in a post-processing step where we use them to get estimates of the individual treatment effects. The regression factor scores are computed based on the full model including both groups and all manifest and latent variables.

The estimates for the individual treatment effects are shown in the output tab labeled "Conditional Effects I". We randomly selected 10 persons from our sample and illustrate the output in Table 3. The first column refers to the treatment variable and then the values of the three manifest covariates are shown for each person. The next seven columns are the estimated factor scores for 
the latent covariates. We then take all covariate values and compute the values of the effect function $\mathrm{g}_{1}(Z)$ which are the estimates for the individual treatment effects shown in the column labeled $\mathrm{g} 1$. As in the Conditional Effects II output discussed earlier, we also get standard errors and estimates for the expected outcome under CBT and under STPP for each person in the dataset. We can also download this data table and use it in our preferred statistical software packages for further analyses or making graphs. To get a better idea about how much variability there is in treatment effects we can look at the estimates themselves or directly go the Plot 4 output (see Figure 4). Figure 4 shows the individual treatment effects for each person. Individual effects in our sample range from approximately -0.5 to 1 . Positive values (red points) indicate an advantage of CBT and negative values (blue points) indicate an advantage of STPP. Notice that CBT seems to have an advantage for more persons in our sample (approximately 75\%), but many estimates are close to zero and nonsignificant since the confidence interval (vertical bars) include zero. In other words, CBT is on average more effective as also indicated by the average effect in the main output (Estimate $=0.176$, $\mathrm{SE}=0.045, \mathrm{p}$-value $=0.000$, effect $\operatorname{size}^{6}=0.373$ ) but there are considerable interindividual differences.

\section{Step 8: Aggregated Effects}

After having discovered that there are interindividual differences in treatment effects we are now interested in explaining these interindividual differences. In particular, we want to investigate the role of each of the covariates in the model for predicting the effectiveness of the treatment and we want to test whether other variables not included in the model can explain some of the heterogeneity in treatment effects. Formally, we consider so-called aggregated effects $E\left(\mathrm{~g}_{1}(Z) \mid W\right)$ that are defined as regressions of the effect function on $W$, where $W$ is a vector of one or more covariates that can either be included in the model or not.

We look at the importance of each covariate for predicting treatment effects. An indication of the importance is the corresponding interaction term in the effect function $g_{1}(Z)$. Table 2 shows a significant interaction of the treatment variable with the second covariate (IIP affiliation, coefficient $\gamma_{102}$ ) and the fourth covariate (pre-treatment LSAS, coefficient $\gamma_{104}$ ). The sign of both interaction terms is positive, meaning that the higher the values of IIP affiliation and pre-treatment LSAS the more the effectiveness of STPP decreases and the effectiveness of CBT increases given everything else is constant. However, the interaction terms do not show the unconditional relationship between the covariates and the treatment effects, because they are conditional on all covariates in the model.

6 The default effect size reported by EffectLiteR is obtained by dividing the average effect by the standard deviation of $Y$ in the control group, i.e., the CBT group. This effect size is slightly higher than the one reported by Leichsenring et al. (2013), because the dependent variable is modeled as latent (without measurement error), the missing data handling is full information maximum likelihood in our analysis, and the model includes more variables, which can lead to variations in estimated effect sizes. 
To examine the unconditional relationship we can look at Plot 3 in the EffectLiteR output panel. Figure 5 shows the regression of the effect function on pre-treatment LSAS which has a positive slope. The regression crosses the zero line at a pre-treatment LSAS value of approximately 1.25. This is the turning point, i.e., STPP has a predicted advantage for lower values of the pre-treatment LSAS score and CBT has a predicted advantage for higher values of the pre-treatment LSAS score. To interpret the value of 1.25 it is helpful to consider the descriptive statistics of the pre-treatment LSAS variable shown in Table 2, i.e., the turning point is estimated approximately at one standard deviation below the mean. To test whether the slope of the regression is significant we must not just regress the effect function on pre-treatment LSAS. This would give us a correct point estimate but severely biased standard errors, because the values of the effect function have been estimated in a previous step. Instead we can use bootstrapping to obtain a confidence interval for the regression slope. EffectLiteR provides an interface for doing this in the Conditional Effects IV tab in the output panel where we can specify the variable $W$ for the aggregated effects regression $E\left(\mathrm{~g}_{1}(Z) \mid W\right)$ $=\alpha_{0}+\alpha_{1} \cdot W$. We use pre-treatment LSAS (lsas.pre) and 200 bootstrap samples to compute the standard errors for the slope $\alpha_{1}$ (Estimate $\left.=0.316, \mathrm{SE}=0.151, \mathrm{p}=0.036\right)$ and indeed find a significant relationship between pre-treatment LSAS and the differential effectiveness of CBT vs. STPP. In a similar way we tested whether the treatment effects depend on IIP affiliation (Estimate $=0.337$, $\mathrm{SE}=0.139, \mathrm{p}=0.015)$ and on the other covariates in the model which are not significant. We can also use the same procedure to test whether other variables that are not included in the model are related to the interindividual differences in treatment effects.

\section{Step 9: Subgroups of Treatment Effects}

In this step we want to examine subgroups of persons who benefit from one or the other treatment. For this purpose we can also use the Conditional Effects IV output and specify a categorical $W$ variable. We tested for example for gender differences in individual treatment effects (Estimate $=0.097, \mathrm{SE}=0.048, \mathrm{p}=0.044)$ indicating significant differences in treatment effects for gender with females having higher treatment effects. In further analyses we looked at differences between responders/non-responders, where responding was defined as a reduction of at least $31 \%$ in social anxiety from pre- to post-treatment in the manifest observed scores (Leichsenring et al., 2013). We did not find significant differences in individual treatment effects between responders and non-responders $($ Estimate $=0.025, \mathrm{SE}=0.044, \mathrm{p}=0.574)$.

Another output that is helpful for looking at subgroups of persons is the Conditional Effects III output. Recall that for the conditional effects given values of covariates (Conditional Effects II output), we had to provide values for all covariates in the model and then the value of the effect function was calculated. In the Conditional Effects III output, it is sufficient to provide values for a 
subset of the covariates in the model. The program then uses a matching procedure based on the Mahalanobis distance to find the subset of persons in our sample that is closest to the selected values. For example, if we specify a pre-treatment LSAS value of two (lsas.t1=2) and one comorbid mental disorder (comorbid=1), the program tries to find the subset of 10 persons (or whichever number of persons the user requests) that are closest to these specified values, i.e., that have a pretreatment social anxiety level around two and one comorbid mental disorder. We can then see the conditional effects output for these persons including the values of all covariates and the estimated treatment effects for each of them. This gives us an impression about how the empirical distribution of treatment effects looks like for persons that are similar with regard to pre-treatment LSAS values and comorbid mental disorders that we specified. In our case, the individual treatment effects for the selected subgroup of 10 persons range from -0.05 to 0.56 . We also get an estimate for the aggregated effect for this subgroup of persons along with a standard error. The aggregated effect is the mean of the ten individual treatment effects and indicates a significant advantage of CBT for this subgroup (Estimate $=0.24, \mathrm{SE}=0.10, \operatorname{ExpOutc} 0=1.21$, ExpOutc1=1.45). If categorical covariates were included in the main output, the matching procedure would use exact matching for the categorical covariates and Mahalanobis distance matching for the continuous covariates.

\section{Additional Features in EffectLiteR}

\section{Multilevel designs}

For studies with multilevel designs, such as psychotherapy studies where patients are nested in therapists or in centers, it can be important to take the multilevel structure of the data into account (e.g., Wampold \& Imel, 2015). In the EffectLiteR approach we can either correct the standard errors of the parameter estimates for the non-independence of the observations or use a multilevel structural equation modeling approach. The former option is useful if all covariates are at the person level and the goal is to get proper standard errors in multilevel designs without explicitly modeling the different levels. The latter option allows for more complex models including manifest and latent covariates at different levels and contextual effects.

The correction of standard errors approach is based on complex survey analysis of structural equation models (Satorra \& Bentler, 1994) to account for multilevel designs. This option is implemented in EffectLiteR both in the syntax and in the graphical user interface in the tab Complex Survey in the input panel where you can specify the cluster variable. This option provides a convenient interface to the lavaan.survey package (Oberski, 2014) which carries out the correction. To test whether we need the correction for the analysis presented in this paper, we first computed the intra-class correlation for all manifest variables in the model and especially for the indicators of the latent dependent variable post-treatment social anxiety. The intra-class correlation 
coefficients were very low (around 0.05) which indicates that the correction is probably not needed. For instructional purposes, we nevertheless fitted the main model with the correction and provide the code in Appendix A to show how to conduct such an analysis. As expected, the point estimates and the standard errors of the parameters were very close to the ones reported in this manuscript. Note that the complex survey correction requires listwise deletion, fixed cells and does not allow for missing values in the therapist variable (which would lead to exclusion of some more observations in the current study). For situations with many missing values in some of the variables in the analysis it can be combined with multiple imputation (Little \& Rubin, 1987).

The multilevel structural equation modeling approach consists of a model for both the patient level and the therapist level and therefore requires a high number of therapists and a substantial amount of variance at the upper level. Mayer et al. (2014) describe how the multilevel SEM approach can be used for an EffectLiteR analysis of average and conditional effects. For the present analysis, we decided not to use such a model, because there are no covariates related to therapists in the analysis and the amount of variance at the upper level is very low as indicated by the low intraclass correlation. The multilevel SEM approach is not yet implemented in the EffectLiteR package, but such models can be specified directly in common SEM software.

\section{Additional Options}

EffectLiteR uses a flexible multigroup structural equation modeling approach based on lavaan (Rosseel, 2012) in the background to estimate all interesting average, conditional and aggregated effects. By relying on lavaan we can easily access many modern statistical techniques that have been adopted or developed in the SEM literature. We already used the full information maximum likelihood estimator to account for missing data (Enders \& Bandalos, 2001) but there are many more options available: We can for example use robust estimators and standard errors based on bootstrapping or sandwich estimators (Yuan \& Bentler, 2000), we can use models with stochastic regressors and group sizes (Mayer \& Thoemmes, 2019), and we can add linear and non-linear constraints in our model (e.g., to restrict interactions or to assume homogeneous residual variances). The graphical user interface allows us to easily specify these additional options in the input panel under the tabs: Options, interactions, user-specified tests. For the exemplary analysis of the SOPHO-NET data presented in this paper, we did not change the default settings for these additional options (except for the missing data handling mentioned above). Further options that are available in the EffectLiteR software include the handling of more than two treatment groups and the inclusion of propensity scores in the analysis (Rosenbaum \& Rubin, 1983; see also Kirchmann et al., 2012, for an application of EffectLiteR with propensity scores in the context of an 
observational study). In Appendix B, we provide a description of all possible options in EffectLiteR that is extracted from the $\mathrm{R}$ help for the main effectLite() function.

\section{Technical details}

The focus of this paper was on explaining how to do a comprehensive analysis of the effects of a treatment from a practical point of view. Therefore, we cannot discuss all of the technical details in this tutorial. Some additional details and general formulas are given in Mayer et al. (2016). The EffectLiteR graphical user interface also provides a lot more output for users interested in these technical details. The tab "Syntax" in the output panel shows both the code that is necessary to run the same EffectLiteR analysis from the command line and also the lavaan input syntax, so that the user could directly use lavaan to estimate the parameters of the multigroup structural equation model with stochastic group sizes. For larger models, the lavaan input syntax can be rather long, because many effects and additional parameters need to be computed, and it would be tedious and error prone to write the code manually. Running either EffectLiteR or lavaan from the command line is an important option for making the analysis easily reproducible. In Appendix A we provide the code that was used in this paper for the main analysis of the SOPHO-NET data. The results tab in the output panel shows the rather long output from the lavaan software package. On top, we see various model fit measures indicating an acceptable model fit for our analysis $\left(\chi^{2}=\right.$ $600.34, d f=384, p=.000 ; \mathrm{CFI}=0.965 ; \mathrm{TLI}=0.954$; RMSEA $=0.052$ ). Thereafter, the results for the two-group structural equation model are given including the group-invariant measurement models, the stochastic group weights, and the regression of the latent outcome variable posttreatment LSAS (1sas.t2) on all manifest and latent covariates in both treatment groups. In the section labeled "Defined Parameters" we see all the new parameters that are computed based on the basic parameters of the two-group model, i.e., all the regression coefficients of the intercept and effect functions, average and conditional effects, and adjusted means that also appear in the main EffectLiteR output, but there, the formatting and appearance is much better readable and userfriendly.

\section{Discussion}

In this paper, we presented a comprehensive analysis of interindividual differences in treatment effects using the EffectLiteR approach. We illustrated the application of this approach with data from a high-quality RCT conducted by the SOPHO-NET to investigate the effectiveness of cognitive-behavioral therapy and short-term psychodynamic psychotherapy for treatment of social phobia. The dataset is typical for the social and behavioral sciences in the sense that many of the constructs assessed are often conceptualized as latent variables that are measured using multiple 
items from questionnaires and psychological tests. Even though there are many different ways to investigate heterogeneity in treatment effects, the EffectLiteR approach is to the best of our knowledge the only approach that explicitly takes latent variables into account. The EffectLiteR approach is conceptually similar to the personalized advantage index, but EffectLiteR offers more options by building on structural equation modeling. For example, it provides standard errors for the estimates of individual effects, it can incorporate latent variables, and it allows to consider many other conditional and aggregated effects that are all well-defined in terms of causality theory. In addition we showed a variety of useful additional information including figures and tables provided by the EffectLiteR software that helps applied researchers to assess interindividual differences in treatment effects.

In our analysis of the SOPHO-NET data, we find considerable interindividual differences, i.e., for some persons CBT is more effective in reducing social anxiety and for other persons in the dataset, STPP is more effective. We presented several figures that show the variability of treatment effects and also allow to examine which (latent) predictors explain interindividual differences in treatment effects. In particular, pre-treatment social anxiety and affiliation are important predictors of which therapy is more effective. We also used various types of subgroup analysis to gain a deeper understanding of the variability in treatment effects using the EffectLiteR approach.

\section{Future Directions}

Two key challenges in analyzing interindividual differences in treatment effects based on latent variable models are the selection of covariates and the specification of interactions and nonlinear terms. Selecting covariates is always a tradeoff between selecting enough covariates to not miss an important source of heterogeneity of treatment effects and selecting too many covariates and get an overfitting model that exaggerates the amount of heterogeneity and yields invalid out-ofsample predictions. To get an adequate picture of the heterogeneity of treatment effects, applied researchers need to be aware of the challenges in selecting covariates and conduct statistical tests whether all relevant covariates have been included. As with selecting covariates there is a similar tradeoff in the specification of interactions and non-linear terms. On the one hand, it is important to estimate enough parameters to fit the model without missing an important characteristic, and, on the other hand, overfitting the model should be avoided. There are multiple statistical approaches that can aid in both the covariate selection process and in the specification of the appropriate functional form including interactions that can in the future be integrated into EffectLiteR. These approaches range from stepwise regression, over lasso and ridge regression, over latent class models, to treebased methods such as random forests and other machine learning techniques to name a couple of popular approaches (see Hastie, Tibshirani \& Friedman, 2009, for a comprehensive overview of 
available methods). However, these methods have been developed in contexts where all variables are observed and only recently, some of these methods have been extended to work with latent variables in a structural equation modeling framework (e.g., ridge or regularized SEM, Jacobucci, Grimm \& McArdle, 2016, SEM trees, Brandmaier, von Oertzen, McArdle \& Lindenberger, 2013, or lasso SEMs, Brandt, Cambria \& Kelava, 2018). Machine learning techniques have also recently been used to model heterogeneity in individual treatment effects. In particular, recursive partitioning (Athey \& Imbens, 2016), random forests (Wager \& Athey, 2017), Bayesian additive regression trees (Green \& Kern, 2010), lasso methods (Athey \& Imbens, 2015), support vector machines (Imai \& Ratkovic, 2013) and ensemble methods (Grimmer, Messing, \& Westwood, 2013) have been proposed as suitable tools to analyze individual treatment effects. Extending these techniques to latent variable models and combining them with the EffectLiteR approach presented in this paper is an important future direction for research.

In many fields, the effect of individual covariates on treatment heterogeneity is probably rather small, and for models with multiple covariates large samples are needed to achieve sufficient power. For example, Luedtke et al. (2019) recommended samples of 300 persons per treatment arm based on simulations using findings from depression research. In order to achieve the necessary power, it will become increasingly important to aggregate data sets and conduct individual participant data meta-analyses (IPD-MA; e.g., Ebert et al., 2018). In this respect, it is important to further develop EffectLiteR in such a way that the nested data structure and the systematically missing values that are typical for IPD-MA can be modeled as well.

\section{References}

Alden, L. E., Wiggins, J. S., \& Pincus, A. L. (1990). Construction of circumplex scales for the Inventory of Interpersonal Problems. Journal of Personality Assessment, 55, 521-536.

Aiken, L. S., West, S. G., \& Reno, R. R. (1991). Multiple regression: Testing and interpreting interactions. Sage.

Ashley, E. A. (2015). The precision medicine initiative: A new national effort. Journal of the American Medical Association, 313, 2119-2120.

Athey, A. \& Imbens, G. (2016) Recursive partitioning for heterogeneous causal effects. Proceedings of the National academy of Sciences of the United States of America, 113, 7353-7360

Athey, S., \& Imbens, G. W. (2015). Machine learning methods for estimating heterogeneous causal effects. Available from https://arxiv.org/abs/1504.01132v3.

Bandalos, D. L. (2018). Measurement theory and applications for the social sciences. New York: Guilford. 
Beck, A. T., Ward, C. H., Mendelson, M., Mock, J., \& Erbaugh, J. (1961). An inventory for measuring depression. Archives of General Psychiatry, 4, 561-571.

Beemer, J., Spoon, K., Fan, J., Stronach, J., Frazee, J. P., Bohonak, A. J., \& Levine, R. A. (2018). Assessing instructional modalities: Individualized treatment effects for personalized learning. Journal of Statistics Education, 26, 31-39.

Brandt, H., Cambria, J., \& Kelava, A. (2018). An adaptive Bayesian lasso approach with spike-andslab priors to identify multiple linear and nonlinear effects in structural equation models. Structural Equation Modeling, 25, 946-960

Brandmaier, A. M., von Oertzen, T., McArdle, J. J., \& Lindenberger, U. (2013). Structural equation model trees. Psychological Methods, 18, 711-86. doi:10.1037/a0030001.

Chang, W., Cheng, J., Allaire, J.J., Xie, Y., \& McPherson, J. (2018). shiny: Web application framework for $R$. R package version 1.1.0. https://CRAN.R-project.org/package=shiny

Cloninger, C. R. (1987). A systematic method for clinical description and classification of personality variants. A proposal. Archives of General Psychiatry, 44, 573-588.

Cohen, Z. D., \& DeRubeis, R. J. (2018). Treatment selection in depression. Annual Review of Clinical Psychology, 14, 209-236. https://doi.org/10.1146/annurev-clinpsy-050817-084746

DeRubeis, R. J., Cohen, Z. D., Forand, N. R., Fournier, J. C., Gelfand, L. A., \& Lorenzo-Luaces, L. (2014). The personalized advantage index: Translating research on prediction into individualized treatment recommendations. A demonstration. PLoS ONE, 9.

Dahabreh, I., Hayward, R., \& Kent, D. (2016). Using group data to treat individuals: Understanding heterogeneous treatment effects in the age of precision medicine and patient-centred evidence. International Journal of Epidemiology, 45, 2184-2193.

Deisenhofer, A.-K., Delgadillo, J., Rubel, J. A., Böhnke, J. R., Zimmermann, D., Schwartz, B., \& Lutz, W. (2018). Individual treatment selection for patients with posttraumatic stress disorder. Depression and Anxiety, 35, 541-550.

Delgadillo, J., Moreea, O., \& Lutz, W. (2016). Different people respond differently to therapy: A demonstration using patient profiling and risk stratification. Behaviour Research and Therapy, 79, 15-22.

Devlieger, I., Mayer, A., \& Rosseel, Y. (2016). Hypothesis testing using factor score regression: A comparison of four methods. Educational and Psychological Measurement, 76, 741770 .

Deusinger, I. M. (1986). Die Frankfurter Selbstkonzeptskalen (FSKN). Göttingen: Hogrefe.

Driessen, E., Smits, N., Dekker, J. J. M., Peen, J., Don, F. J., Kool, S., Westra, D., Hendriksen, M., Cuijpers, P., \& Van, H. L. (2016). Differential efficacy of cognitive behavioral therapy and psychodynamic therapy for major depression: a study of prescriptive factors. Psychological 
Medicine, 46, 731-744.

Ebert, D. D., Buntrock, C., Reins, J. A., Zimmermann, J., \& Cuijpers, P. (2018). Efficacy and moderators of psychological interventions in treating subclinical symptoms of depression and preventing major depressive disorder onsets: Protocol for an individual patient data meta-analysis of randomised controlled trials. BMJ Open, 8, e018582.

Enders, C. K., \& Bandalos, D. L. (2001). The relative performance of full information maximum likelihood estimation for missing data in structural equation models. Structural Equation Modeling, 8, 430-457.

Fraley, R. C., Waller, N. G., \& Brennan, K. A. (2000). An item-response theory analysis of selfreport measures of adult attachment. Journal of Personality and Social Psychology, 78, $350-365$.

First, M. B., Gibbon, M., Spitzer, R. L., Williams, J. B. W., \& Benjamin, L. S. (1997). Structured clinical interview for DSM-IV axis II personality disorders (SCID-II). Washington, DC: American Psychiatric Press

First, M. B., Spitzer, R. L., Gibbon, M., \& Williams, J. B. W. (2002). Structured clinical interview for DSM-IV-TR axis I disorders (SCID-I). New York, NY: Biometrics Research, New York State Psychiatric Institute.

Fournier, J. C., DeRubeis, R. J., Shelton, R. C., Hollon, S. D., Amsterdam, J. D., \& Gallop, R. (2009). Prediction of response to medication and cognitive therapy in the treatment of moderate to severe depression. Journal of Consulting and Clinical Psychology, 77, 775-787.

Green, D. \& Kern, H. (2010). Modeling heterogeneous treatment effects in large-scale experiments using bayesian additive regression trees. Proceedings of the annual summer meeting of the Society of Political Methodology.

Grimmer, J.; Messing, S. \& Westwood, S. (2013). Estimating heterogeneous treatment effects and the effects of heterogeneous treatments with ensemble methods. Political Analysis, $25,1-22$.

Hamburg, M. A., \& Collins, F. S. (2010). The path to personalized medicine. New England Journal of Medicine, 363, 301-304.

Hastie, T., Tibshirani, R., \& Friedman, J. (2009). The elements of statistical learning. New York: Springer series in statistics.

Heckman, J. J., \& Vytlacil, E. (2005). Structural equations, treatment effects, and econometric policy evaluation. Econometrica, 73, 669-738.

Holland, P. W. (1986). Statistics and causal inference. Journal of the American Statistical Association, 81, 945-960.

Holland, P. W. (1988). Causal inference, path analysis, and recursive structural equation models. 
Sociological Methodology, 18, 449-484.

Hoyer, J., Wiltink, J., Hiller, W., Miller, R., Salzer, S., Sarnowsky, S., Stangier, U., ...Leibing, E. (2016). Baseline patient characteristics predicting outcome and attrition in cognitive therapy for social phobia: Results from a large multicentre trial. Clinical Psychology \& Psychotherapy, 23, 35-46.

Huibers, M. J. H., Cohen, Z. D., Lemmens, L. H. J. M., Arntz, A., Peeters, F. P. M. L., Cuijpers, P., \& DeRubeis, R. J. (2015). Predicting optimal outcomes in cognitive therapy or interpersonal psychotherapy for depressed individuals using the personalized advantage index approach. PLoS ONE, 10, e0140771.

Imai, K. \& Strauss, A. (2011). Estimation of heterogeneous treatment effects from randomized experiments, with application to the optimal planning of the get-out-the-vote campaign. Political Analysis, 19, 1-19.

Imai, K. \& Ratkovic, M. (2013). Estimating treatment effect heterogeneity in randomized program evaluation. Annals of Applied Statistics, 7, 443-470.

Imbens, G. \& Rubin, D.B. (2015). Causal inference for statistics, social, and biomedical sciences. Cambridge University Press.

Jaccard, J., \& Wan, C. K. (1995). Measurement error in the analysis of interaction effects between continuous predictors using multiple regression: Multiple indicator and structural equation approaches. Psychological Bulletin, 117, 348-357.

Jacobucci, R., Grimm, K. J., \& McArdle, J. J. (2016). Regularized structural equation modeling. Structural Equation Modeling, 23, 555-566.

Kazdin, A. E. (2014). Moderators, mediators, and mechanisms of change in psychotherapy. In: W. Lutz \& S. Knox (Eds.). Quantitative and qualitative methods in psychotherapy (pp. 87-101). New York: Routledge.

Kessler, R. C. (2018). The potential of predictive analytics to provide clinical decision support in depression treatment planning. Current Opinion in Psychiatry, 31, 32-39.

Leichsenring, F., Hoyer, J., Beutel, M., Herpertz, S., Hiller, W., Irle, E., Joraschky, P., ...Leibing, E. (2009). The social phobia psychotherapy research network. The first multicenter randomized controlled trial of psychotherapy for social phobia: Rationale, methods and patient characteristics. Psychotherapy and Psychosomatics, 78, 35-41.

Leichsenring, F., Salzer, S., Beutel, M. E., Herpertz, S., Hiller, W., Hoyer, J., Huesing, J., ... Leibing, E. (2013). Psychodynamic therapy and cognitive-behavioral therapy in social anxiety disorder: A multicenter randomized controlled trial. American Journal of Psychiatry, 170, 759-767. 
Leichsenring, F., Salzer, S., Beutel, M. E., Herpertz, S., Hiller, W., Hoyer, J., Huesing, J., ... Leibing, E. (2014). Long-term outcome of psychodynamic therapy and cognitive-behavioral therapy in social anxiety disorder. American Journal of Psychiatry, 171, 1074-1082.

Li, M. \& Tobias, J. (2011) Bayesian inference in a correlated random coefficients model: Modeling causal effect heterogeneity with an application to heterogeneous returns to schooling. Journal of Econometrics, 162, 345-361

Liebowitz, M. R. (1987). Social phobia. Modern Problems of Pharmacopsychiatry, 22, 141-173.

Little, T. D., Rhemtulla, M., Gibson, K., \& Schoemann, A. M. (2013). Why the items versus parcels controversy needn't be one. Psychological Methods, 18, 285-300.

Little, R. J. A., Rubin, D. B. (1987). Statistical analysis with missing data. John Wiley \& Sons, New York.

Luedtke, A., Sadikova, E., \& Kessler, R. C. (2019). Sample Size Requirements for Multivariate Models to Predict Between-Patient Differences in Best Treatments of Major Depressive Disorder. Clinical Psychological Science, 24, doi:216770261881546.

Lutz, W., Leach, C., Barkham, M., Lucock, M., Stiles, W. B., Evans, C., Noble, R., ...Iveson, S. (2005). Predicting change for individual psychotherapy clients on the basis of their nearest neighbors. Journal of Consulting and Clinical Psychology, 73, 904-913.

Manski, C. F. (1995). Identification problems in the social sciences. Harvard University Press.

Marsh, H. W., Lüdtke, O., Nagengast, B., Morin, A. J., \& Von Davier, M. (2013). Why item parcels are (almost) never appropriate: Two wrongs do not make a right-Camouflaging misspecification with item parcels in CFA models. Psychological Methods, 18, 257-284.

Mayer, A., Dietzfelbinger, L., Rosseel, Y., \& Steyer, R. (2016). The EffectLiteR approach for analyzing average and conditional effects. Multivariate Behavioral Research, 51, $374-391$.

Mayer, A., Nagengast, B., Fletcher, J., \& Steyer, R. (2014). Analyzing average and conditional effects with multigroup multilevel structural equation models. Frontiers in Psychology, 5, Article 304.

Mayer, A. \& Thoemmes, F. (2019). Analysis of variance methods with stochastic group sizes. Multivariate Behavioral Research, 54, 543-554.

Mayer, A., Umbach, N., Flunger, B., \& Kelava, A. (2017). Effect analysis using nonlinear structural equation mixture modeling. Structural Equation Modeling, 24, 556-570.

Meredith, W. (1993). Measurement invariance, factor analysis and factorial invariance. Psychometrika, 58, 525-543.

Morgan, S. (2001). Counterfactuals, causal effect heterogeneity, and the catholic school effect on learning. Sociology of Education, 74, 341-374. 
Niles, A. N., Loerinc, A. G., Krull, J. L., Roy-Byrne, P., Sullivan, G., Sherbourne, C. D., Bystritsky, A., \& Craske, M. G. (2017). Advancing personalized medicine: Application of a novel statistical method to identify treatment moderators in the coordinated anxiety learning and management study. Behavior Therapy, 48, 490-500.

Oberski, D. (2014). lavaan.survey: An R package for complex survey analysis of structural equation models. Journal of Statistical Software, 57, 1-27.

R Core Team (2017). $R$ : A language and environment for statistical computing. R Foundation for Statistical Computing, Vienna, Austria. URL https://www.R-project.org/.

Robins, J. M. (1999). Marginal structural models versus structural nested models as tools for causal inference. In M. E. Halloran and D. A. Berry (Eds.), Statistical Models in Epidemiology, the Environment and Clinical Trials (pp. 95-134). Springer, New York.

Rosenbaum, P. R., \& Rubin, D. B. (1983). The central role of the propensity score in observational studies for causal effects. Biometrika, 70, 41-55.

Rosseel, Y. (2012). lavaan: An R package for structural equation modeling and more. Version $0.5-$ 12. Journal of Statistical Software, 48, 1-36.

Rubin, D. B. (1974). Estimating causal effects of treatments in randomized and nonrandomized studies. Journal of Educational Psychology, 66, 688-701.

Satorra, A., \& Bentler, P. M. (1994). Corrections to test statistics and standard errors in covariance structure analysis. In A. von Eye \& C. C. Clogg (Eds.), Latent variables analysis: Applications for developmental research (pp. 399-419). Thousand Oaks, CA: Sage.

Simon, G. \& Perlis, R. (2010). Personalized medicine for depression: Can we match patients with treatments? American Journal of Psychiatry, 167, 1445-1455.

Snow, R. E. (1991). Aptitude-treatment interaction as a framework for research on individual differences in psychotherapy. Journal of Consulting and Clinical Psychology, 59, 205-216.

Steyer, R. (2005). Analyzing individual and average causal effects via structural equation models. Methodology, 1, 39-54.

Steyer, R., Mayer, A., \& Fiege, C. (2014). Causal inference on total, direct, and indirect effects. In A. C. Michalos (Ed.), Encyclopedia of Quality of Life and Well-Being Research (pp. 606-631). Dordrecht, Netherlands: Springer.

Steyer, R., Gabler, S., von Davier, A. A., Nachtigall, C., \& Buhl, T. (2000). Causal regression models I: Individual and average causal effects. Methods of Psychological Research Online, 5, 39-71.

Steyer, R., \& Partchev, I. (2008). EffectLite: 3.0-Software und user's manual. University of Jena. Strauss, B., Koranyi, S., Altmann, U., Nolte, T., Beutel, M. E., Wiltink, J., Herpertz, S., ... Kirchmann, H. (2017). Partner-related attachment as a moderator of outcome in patients 
with social anxiety disorder-a comparison between short-term cognitive-behavioral and psychodynamic therapy. Psychotherapy, 54, 339-350.

Pearl, J. (2009). Causality: Models, reasoning, and inference ( $2^{\text {nd }}$ ed.). Cambridge, UK: Cambridge University Press.

Wager, S. \& Athey, S. (2017). Estimation and inference of heterogeneous treatment effects using random forests. Journal of the American Statistical Association, doi:10.1080/01621459.2017.1319839.

Wampold, B. E., \& Imel, Z. E. (2015). The great psychotherapy debate: The evidence for what makes psychotherapy work ( $2^{\text {nd }}$ ed.). New York: Routledge Taylor \& Francis.

Webb, C. A., Trivedi, M. H., Cohen, Z. D., Dillon, D. G., Fournier, J. C., Goer, F., Fava, M., McGrath, P. J., Weissman, M., Parsey, R., Adams, P., Trombello, J. M., Cooper, C., Deldin, P., Oquendo, M. A., McInnis, M. G., Huys, Q., Bruder, G., Kurian, B. T., Jha, M., DeRubeis, R. J., \& Pizzagalli, D. A. (in press). Personalized prediction of antidepressant v. placebo response: evidence from the EMBARC study. Psychological Medicine.

Wiltink, J., Hoyer, J., Beutel, M. E., Ruckes, C., Herpertz, S., Joraschky, P., Koranyi, S., ... Leichsenring, F. (2016). Do patient characteristics predict outcome of psychodynamic psychotherapy for social anxiety disorder? PLOS ONE, 11, e0147165.

Winship, C., \& Morgan, S. L. (1999). The estimation of causal effects from observational data. Annual Review of Sociology, 25, 659-707.

Yuan, K. H., \& Bentler, P. M. (2000). Three likelihood based methods for mean and covariance structure analysis with nonnormal missing data. Sociological Methodology, 30, 165-200.

Xie, Y.; Brand, J. \& Jann, B. (2012). Estimating heterogeneous treatment effects with observational data. Sociological Methodology, 42, 314-347 
Table 1

Regression coefficients for the final model with ten covariates and all treatment by covariate interactions.

\begin{tabular}{|c|c|c|c|c|c|}
\hline Coefficient & Variable & Estimate & SE & Est./SE & p-value \\
\hline \multicolumn{6}{|c|}{ Intercept function $\mathrm{g}_{0}(Z)$} \\
\hline$\gamma_{00}$ & & 0.758 & 0.584 & 1.299 & 0.194 \\
\hline$\gamma_{01}$ & $Z_{1}$ & 0.109 & 0.038 & 2.866 & 0.004 \\
\hline$\gamma_{02}$ & $Z_{2}$ & -0.296 & 0.099 & -3.000 & 0.003 \\
\hline$\gamma_{03}$ & $Z_{3}$ & 0.089 & 0.083 & 1.067 & 0.286 \\
\hline$\gamma_{04}$ & $\xi_{1}\left(Z_{4}\right)$ & 0.408 & 0.087 & 4.681 & 0.000 \\
\hline$\gamma_{05}$ & $\xi_{2}\left(Z_{5}\right)$ & -0.135 & 0.153 & -0.884 & 0.376 \\
\hline$\gamma_{06}$ & $\xi_{3}\left(Z_{6}\right)$ & 0.062 & 0.035 & 1.783 & 0.075 \\
\hline$\gamma_{07}$ & $\xi_{4}\left(Z_{7}\right)$ & -0.094 & 0.042 & -2.243 & 0.025 \\
\hline$\gamma_{08}$ & $\xi_{5}\left(Z_{8}\right)$ & 0.274 & 0.378 & 0.725 & 0.468 \\
\hline$\gamma_{09}$ & $\xi_{6}\left(Z_{9}\right)$ & -0.016 & 0.091 & -0.175 & 0.861 \\
\hline$\gamma_{010}$ & $\xi_{7}\left(Z_{10}\right)$ & -0.123 & 0.075 & -1.636 & 0.102 \\
\hline \multicolumn{6}{|c|}{ Effect function $g_{1}(Z)$} \\
\hline$\gamma_{10}$ & $X$ & -1.543 & 0.838 & -1.842 & 0.065 \\
\hline$\gamma_{11}$ & $X \cdot Z_{1}$ & -0.041 & 0.054 & -0.751 & 0.453 \\
\hline$\gamma_{12}$ & $X \cdot Z_{2}$ & 0.319 & 0.138 & 2.314 & 0.021 \\
\hline$\gamma_{13}$ & $X \cdot Z_{3}$ & -0.168 & 0.129 & -1.306 & 0.191 \\
\hline$\gamma_{14}$ & $X \cdot \xi_{1}$ & 0.335 & 0.149 & 2.242 & 0.025 \\
\hline$\gamma_{15}$ & $X \cdot \xi_{2}$ & 0.344 & 0.215 & 1.597 & 0.110 \\
\hline$\gamma_{16}$ & $X \cdot \xi_{3}$ & 0.026 & 0.055 & 0.461 & 0.645 \\
\hline$\gamma_{17}$ & $X \cdot \xi_{4}$ & 0.024 & 0.059 & 0.411 & 0.681 \\
\hline$\gamma_{18}$ & $X \cdot \xi_{5}$ & -0.749 & 0.616 & -1.216 & 0.224 \\
\hline$\gamma_{19}$ & $X \cdot \xi_{6}$ & 0.149 & 0.125 & 1.192 & 0.233 \\
\hline$\gamma_{110}$ & $X \cdot \xi_{7}$ & 0.178 & 0.107 & 1.664 & 0.096 \\
\hline
\end{tabular}

Note. Variables included in the analysis are $\mathrm{X}$ (treatment variable, $0=\mathrm{CBT}$, $1=\mathrm{STPP}$ ), $Z_{1}$ (comorbid mental disorders), $Z_{2}$ (IIP affiliation), $Z_{3}$ (IIP dominance), $\xi_{1}=Z_{4}$ (pre-treatment LSAS), $\xi_{2}=Z_{5}$ (BDI), $\xi_{3}=Z_{6}$ (ECR anxiety),

$\xi_{4}=Z_{7}$ (ECR avoidance), $\xi_{5}=Z_{8}$ (TPQ harm avoidance), $\xi_{6}=Z_{9}$ (TOSCA shame), $\xi_{7}=Z_{10}$ (FSCN self-esteem). 
Table 2

Descriptive statistics for continuous manifest and latent covariates.

\begin{tabular}{llccc}
\hline \multicolumn{1}{c}{ Covariate } & \multicolumn{1}{c}{ Variable Name } & Type & Mean & SD \\
\hline Comorbid mental disorder & $Z_{1}=$ comorbid & manifest & 0.921 & 0.915 \\
IIP affiliation & $Z_{2}=$ iip.lov & manifest & -0.061 & 0.385 \\
IIP dominance & $Z_{3}=$ iip.dom & manifest & -0.802 & 0.425 \\
Pre-treatment LSAS & $\xi_{1}=$ lsas.lt1 & latent & 1.650 & 0.441 \\
BDI & $\xi_{2}=$ bdi.lt1 & latent & 0.708 & 0.418 \\
ECR anxiety & $\xi_{3}=$ ecr.anx.lt1 & latent & 3.409 & 1.193 \\
ECR avoidance & $\xi_{4}=$ ecr.avoi.lt1 & latent & 2.871 & 1.071 \\
TPQ harm avoidance & $\xi_{5}=$ hal & latent & 0.669 & 0.132 \\
TOSCA shame & $\xi_{6}=$ tosca.shame.t1 & latent & 3.248 & 0.627 \\
FSCN self-esteem & $\xi_{7}=$ fskn.se.t1 & latent & 3.948 & 0.942 \\
\hline
\end{tabular}


Table 3

Conditional Effects I Output.

\begin{tabular}{ccccccccccccccc}
\hline \multicolumn{1}{c}{ Covariates } & \multicolumn{1}{c}{ Effects } \\
$\mathrm{X}$ & $Z_{1}$ & $Z_{2}$ & $Z_{3}$ & $\xi_{1}$ & $\xi_{2}$ & $\xi_{3}$ & $\xi_{4}$ & $\xi_{5}$ & $\xi_{6}$ & $\xi_{7}$ & $g_{1}(Z)$ & $S E$ & $\begin{array}{c}\text { Exp } \\
\text { Exp }\end{array}$ \\
& & & & & & & & & & & & & Out0 & Out1 \\
\hline 0 & 0 & -0.39 & -1.10 & 1.17 & 0.63 & 2.82 & 2.59 & 0.63 & 2.15 & 4.42 & -0.10 & 0.15 & 0.69 & 0.59 \\
1 & 0 & -0.33 & -0.47 & 2.05 & 1.42 & 4.81 & 3.77 & 0.95 & 4.19 & 2.41 & 0.17 & 0.18 & 1.30 & 1.46 \\
0 & 1 & -0.01 & 0.10 & 1.85 & 0.93 & 3.96 & 3.55 & 0.68 & 3.43 & 3.35 & 0.12 & 0.14 & 1.14 & 1.26 \\
0 & 1 & -0.69 & -0.78 & 0.78 & 0.53 & 1.77 & 1.07 & 0.73 & 2.76 & 4.67 & -0.46 & 0.22 & 0.84 & 0.38 \\
1 & 0 & -0.26 & -1.38 & 1.48 & 1.29 & 4.28 & 3.21 & 0.77 & 4.01 & 2.19 & 0.15 & 0.17 & 0.98 & 1.13 \\
0 & 1 & 0.54 & -0.90 & 1.67 & 0.60 & 3.87 & 3.97 & 0.77 & 3.74 & 2.90 & 0.20 & 0.17 & 0.89 & 1.08 \\
0 & 0 & -0.01 & -0.38 & 1.21 & 0.22 & 2.50 & 1.57 & 0.48 & 2.83 & 5.24 & 0.10 & 0.11 & 0.64 & 0.74 \\
1 & 2 & 0.19 & -1.41 & 2.28 & 1.59 & 4.90 & 4.87 & 0.88 & 4.39 & 2.11 & 0.60 & 0.14 & 1.27 & 1.87 \\
1 & 3 & 0.24 & -0.96 & 1.28 & 0.00 & 1.81 & 1.57 & 0.38 & 1.55 & 5.49 & 0.01 & 0.25 & 0.82 & 0.83 \\
1 & 0 & -0.11 & -0.63 & 1.64 & 0.47 & 3.76 & 2.97 & 0.73 & 3.35 & 4.56 & 0.18 & 0.11 & 0.88 & 1.06 \\
\hline
\end{tabular}

Note. Variables included in the analysis are $\mathrm{X}$ (treatment variable, $0=\mathrm{CBT}, 1=\mathrm{STPP}$ ), $Z_{1}$ (comorbid mental disorders), $Z_{2}$ (IIP affiliation), $Z_{3}$ (IIP dominance), $\xi_{1}=Z_{4}$ (pre-treatment LSAS), $\xi_{2}=Z_{5}$ (BDI), $\xi_{3}=Z_{6}$ (ECR anxiety), $\xi_{4}=Z_{7}$ (ECR avoidance), $\xi_{5}=Z_{8}$ (TPQ harm avoidance), $\xi_{6}=Z_{9}$ (TOSCA shame), $\xi_{7}=Z_{10}$ (FSCN self-esteem). The table shows the individual treatment effects $g_{1}(Z)$ with standard errors $(S E)$ and expected outcomes under CBT (ExpOut0) and STPP (ExpOut1). 


\section{EffectLiteR}

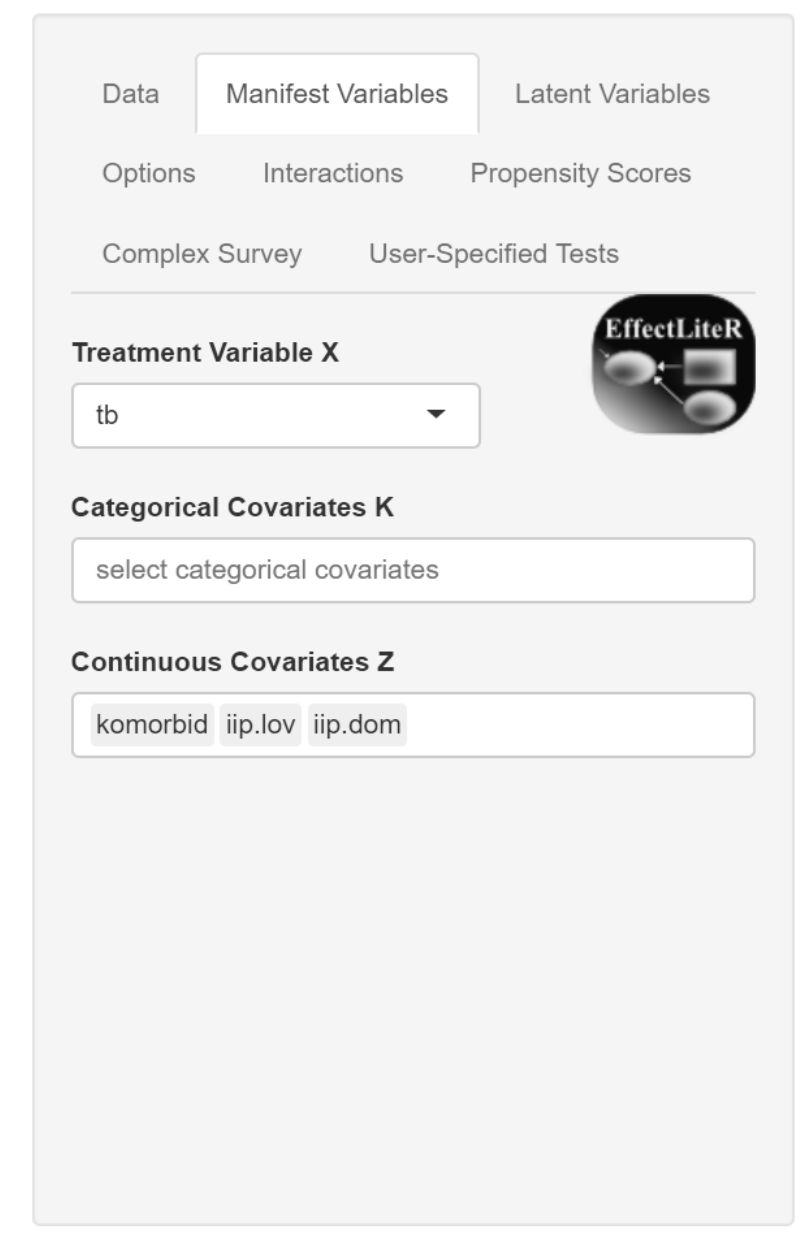

\section{EffectLiteR}

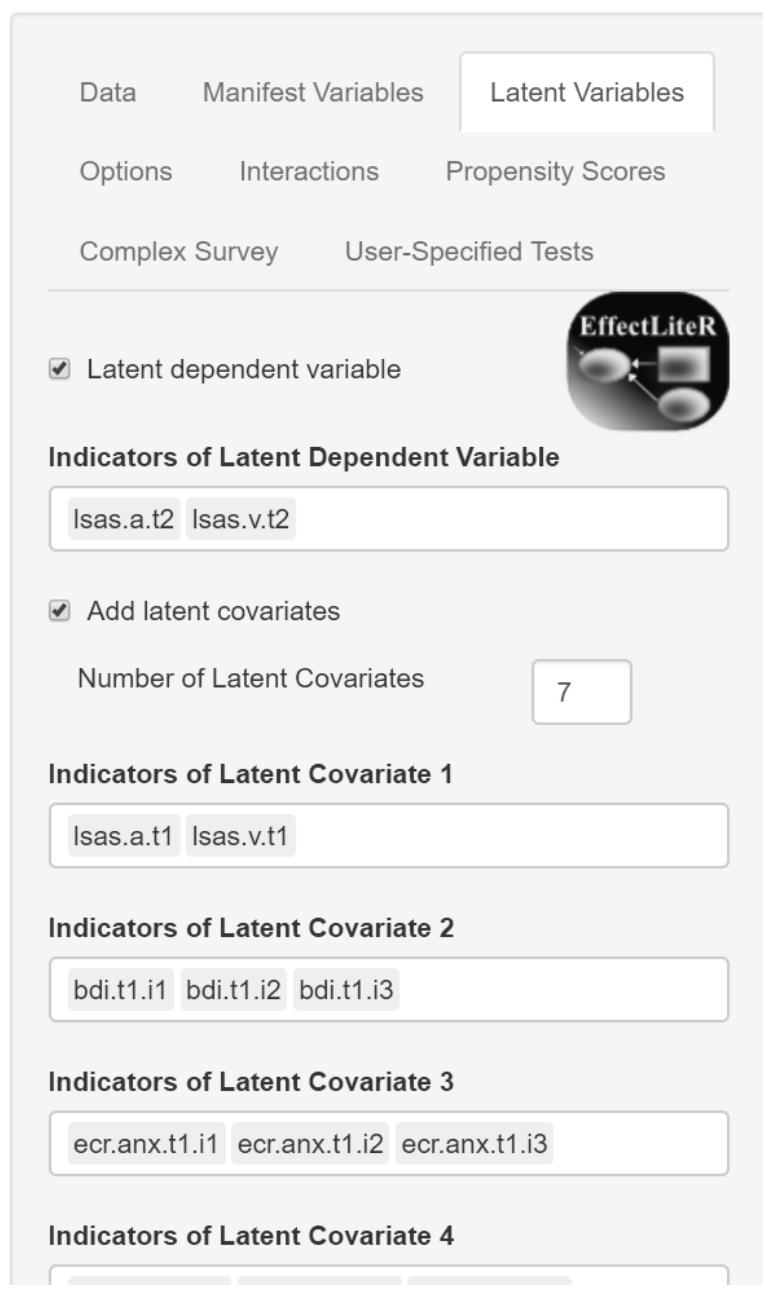

Figure 1. Specification of manifest and latent variables in the EffectLiteR graphical user interface for the final model with ten covariates. 


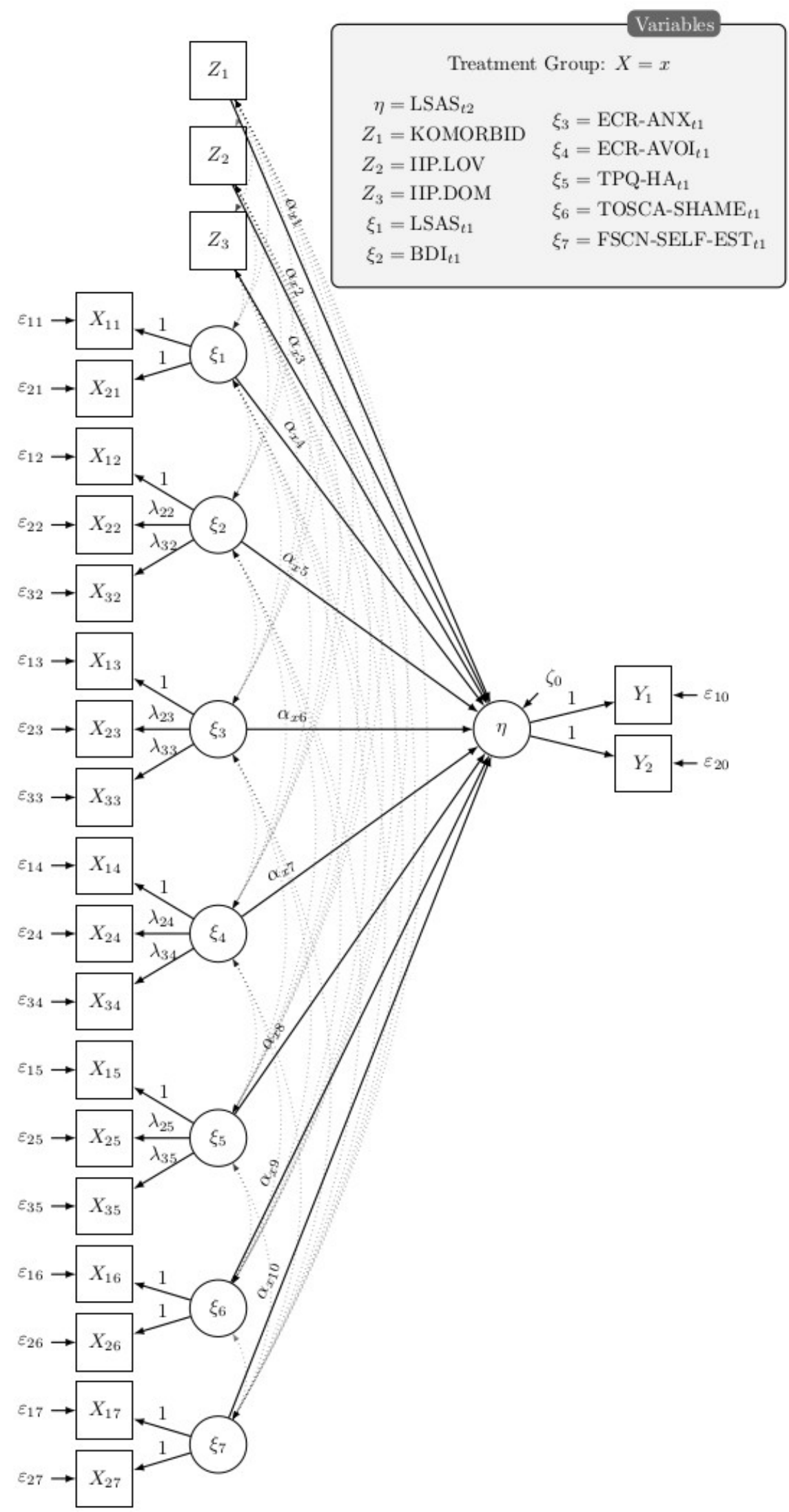

Figure 2. Path diagram of the regression of $\eta$ on the manifest covariates $Z_{1}$ to $Z_{3}$ and the latent covariates $\xi_{1}$ to $\xi_{7}$ in both the CBT group and in the STPP group. Covariances between all independent variables are indicated by semitransparent double-headed arrows. 
Individual Treatment Effects with Latent Variables

Data EffectLiteR Syntax Results Conditional Effects I Conditional Effects II Conditional Effects III

Conditional Effects IV $\quad$ User-Specified Tests $\quad$ Plot $1 \quad$ Plot $2 \quad$ Plot $3 \quad$ Plot 4

Conditional effects for user specified values of categorical and continuous covariates.

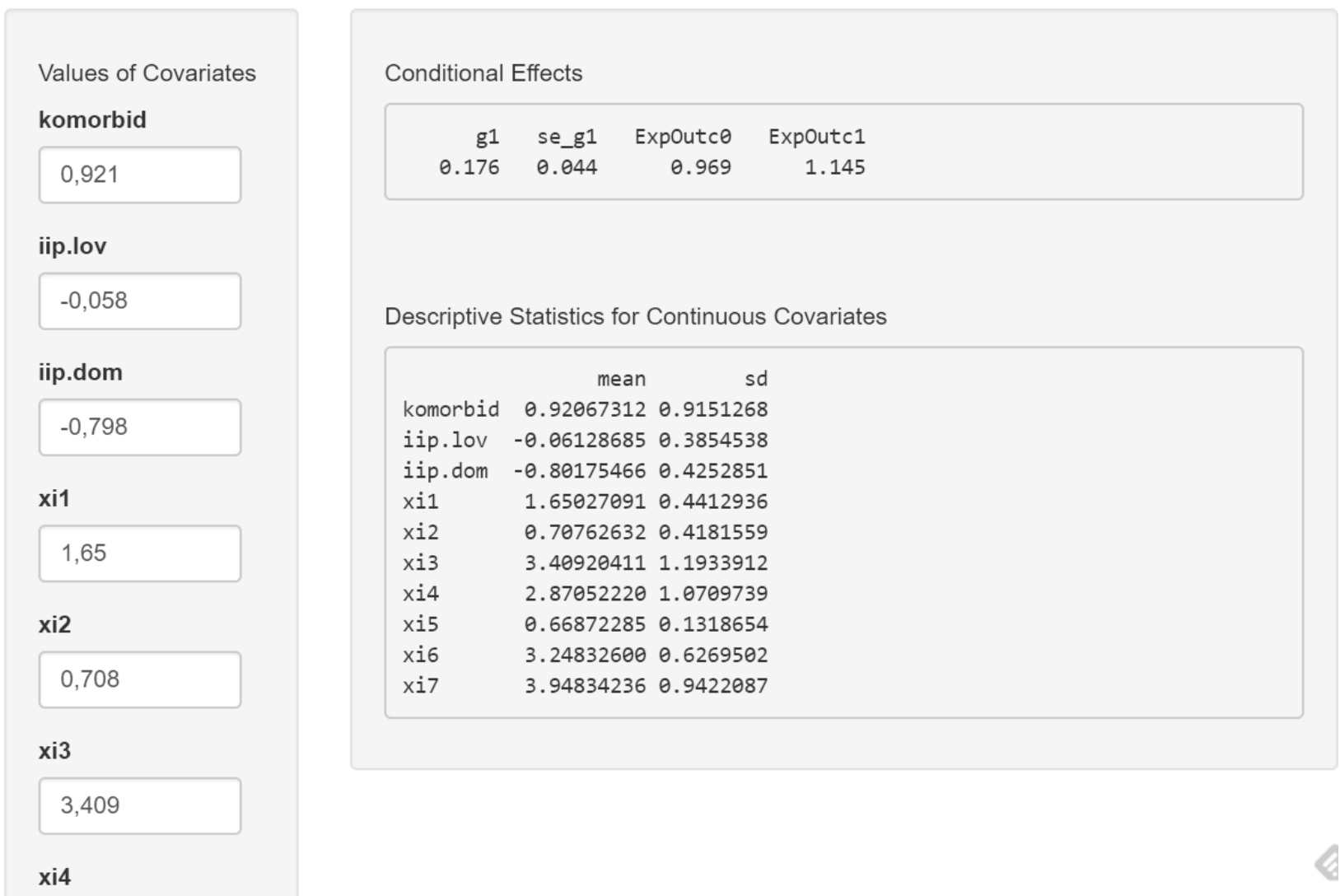

Figure 3. Conditional Effects II output tab. This output shows the conditional treatment effect $\left(\mathrm{g}_{1}\right)$ for a person with covariate values as specified on the left hand side. In addition, the standard error (se_g $\mathrm{g}_{1}$ ) and the expected outcomes under CBT (ExpOutc0) and under STPP (ExpOutc0) are depicted. At the bottom, the means and standard deviations of all covariates are shown. 


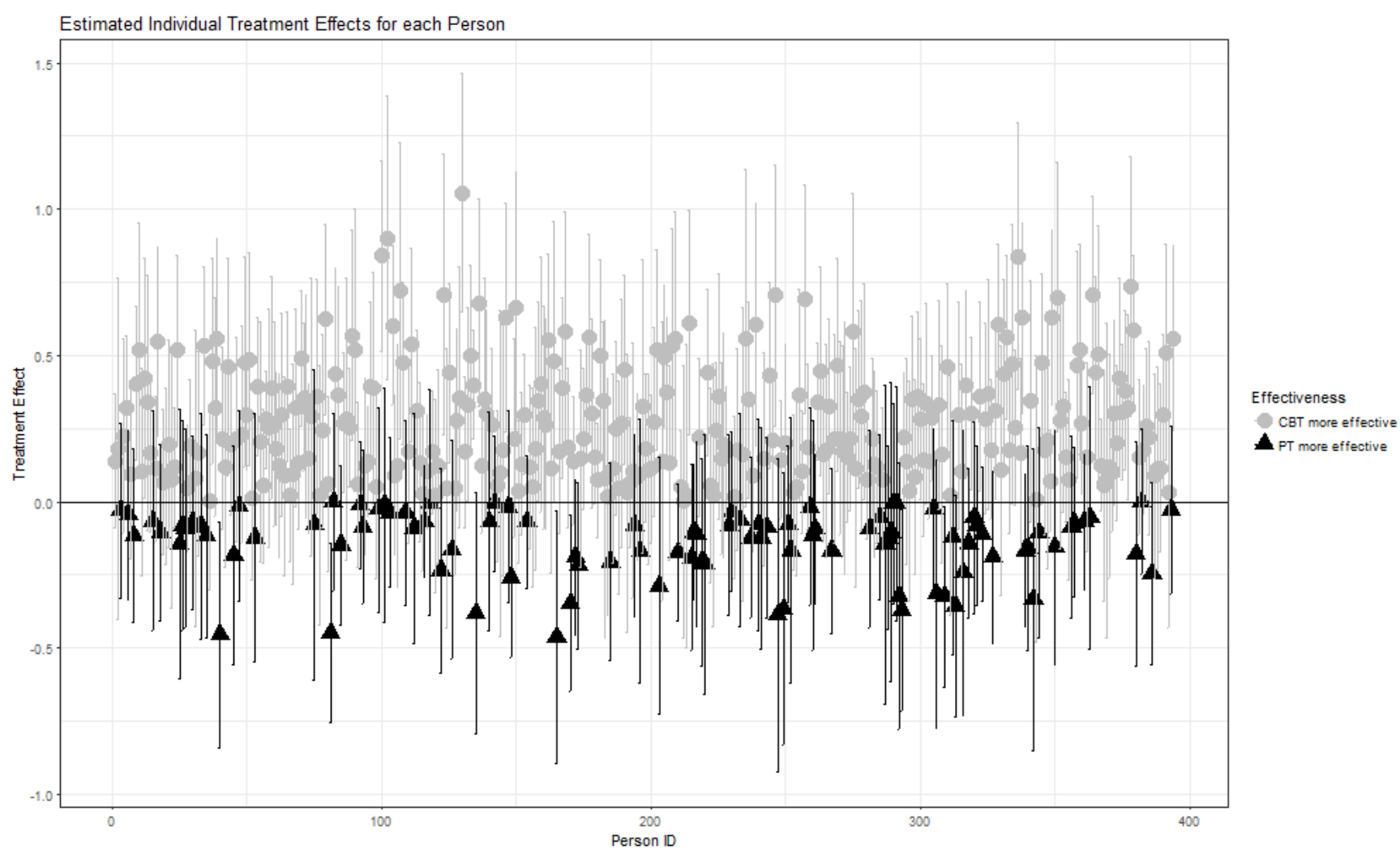

Figure 4. Estimated individual treatment effects for each person in the sample. The effects are comparisons between STPP and CBT, i.e., positive values indicate an advantage of CBT and negative values indicate an advantage of STPP. 95\% confidence intervals are shown around the point estimates. 


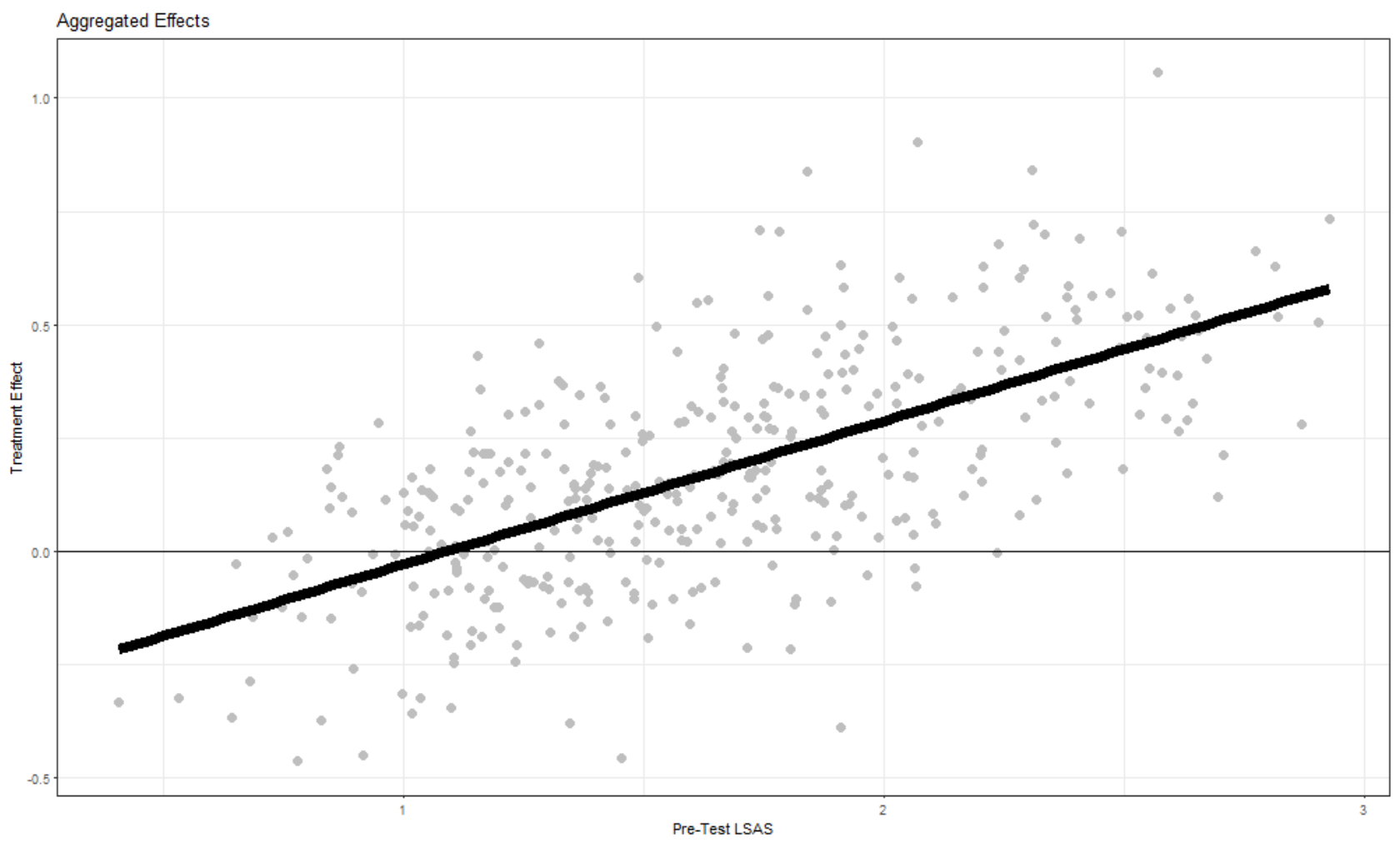

Figure 5. Aggregated effects plot for pre-treatment LSAS. The relationship between the treatment effects (y-axis) and the pre-treatment LSAS values (x-axis) is depicted. The treatment effects are comparisons between STPP and CBT, i.e., positive values indicate an advantage of CBT and negative values indicate an advantage of STPP. 


\section{Supplemental Materials}

\section{Appendix A: Software Code}

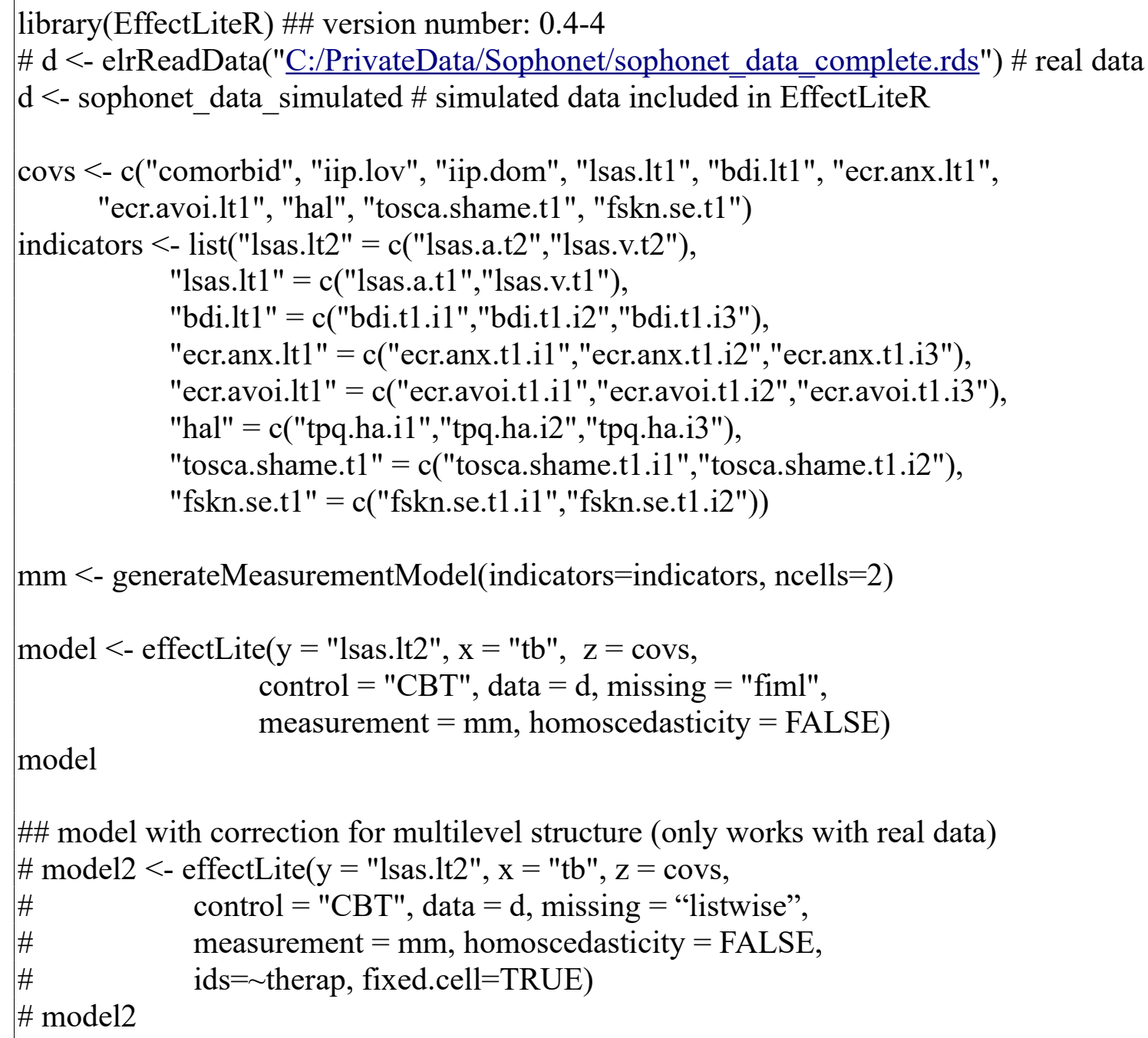




\section{Appendix B: Options in effectLite()}

$\mathrm{y}$

$\mathrm{x}$

$\mathrm{k}$

Z

data

method

control

measurement

fixed.cell

fixed.z

missing

se

syntax.only

interactions

homoscedas-

ticity

test.stat

propscore
Dependent variable (character string). Can be the name of a manifest variable or of a latent variable.

Treatment variable (character string) treated as categorical variable.

Vector of manifest variables treated as categorical covariates (character vector).

Vector of continuous covariates (character vector). Names of both manifest and latent variables are allowed.

A data frame.

Can be one of c("sem","lm") and indicates which function is used to fit the model.

Value of $x$ that is used as control group. If "default", takes the first entry of as.factor(x).

Measurement model. The measurement model is lavaan syntax (character string), that will be appended before the automatically generated lavaan input. It can be used to specify a measurement for a latent outcome variable and/or latent covariates. See also the example and generateMeasurementModel.

logical. If FALSE (default), the group sizes are treated as stochastic rather than fixed.

logical. If FALSE (default), the continuous covariates are treated as stochastic rather than fixed.

Missing data handling. Will be passed on to sem or ignored for method="lm".

Type of standard errors. Will be passed on to sem or ignored for method="lm".

logical. If TRUE, only syntax is returned and the model will not be estimated.

character. Indicates the type of interaction. Can be one of "all (all interactions), "2way" (only two-way interactions), "X:K,X:Z" (only X:K and X:Z interactions), "X:K" (only $\mathrm{X}: \mathrm{K}$ interactions), "X:Z" (only X:Z interactions), "none" (no treatment by covariate interactions, but potentially interactions between categorical and continuous covariates), or "no" (no interactions at all).

logical. If TRUE, residual variances of the dependent variable are assumed to be homogeneous across cells.

character. Can be one of c("default","Chisq","Ftest") and indicates the statistic used for the hypothesis tests. The tests are either based on the large sample Chi-Squared statistic (Wald tests) or the finite sample F statistic with approximate F distribution. The default setting for method="sem" is"Chisq" and the default setting for method="lm" is "Ftest".

Vector of covariates (character vector) that will be used to compute (multiple) propensity scores based on a multinomial regression without interactions. Alternatively, the user can specify a formula with the treatment variable as dependent variable for more control over the propensity score model.

ids
Formula specifying cluster ID variables. Will be passed on to lavaan.survey. See svydesign for details. 
weights Formula to specify sampling weights. Currently only one weight variable is supported. Will be passed on to lavaan.survey. See svydesign for details. Note: Only use weights if you know what you are doing. For example, some conditional treatment effects may require different weights than average effects.

add Character string that will be pasted at the end of the generated lavaan syntax. Can for example be used to add additional (in-) equality constraints or to compute userdefined conditional effects.

Further arguments passed to $\underline{\text { sem. }}$. 